\title{
Stress and strain relaxation in magnesium AZ31 rolled plate: in-situ neutron measurement and elastic viscoplastic polycrystal modeling
}

\author{
H. Wang ${ }^{\text {a, }}$, B. Clausen ${ }^{\text {a }}$, L. Capolungo ${ }^{b}$, I.J. Beyerlein ${ }^{c}$, J. Wang ${ }^{\text {a }}$, C.N. Tomé ${ }^{a}$ \\ ${ }^{a}$ Materials Science and Technology Division, Los Alamos National Laboratory, Los Alamos, \\ NM, 87545, USA \\ ${ }^{\mathrm{b}}$ George Woodruff School of Mechanical Engineering, Georgia Institute of Technology \\ and UMI 2958 Georgia Tech-CNRS, 57070 Metz, France \\ ${ }^{c}$ Theoretical Division, Los Alamos National Laboratory, Los Alamos, NM, 87545, USA
}

\begin{abstract}
Continuous mechanical tests with strain holds (stress relaxation) and with stress holds (strain relaxation) are performed simultaneously with in-situ neutron measurements to analyze the mechanisms of stress and strain relaxation in $\mathrm{Mg}$ AZ31 rolled plate. A dislocation activity based constitutive model, accounting for internal stress statistical distributions, is proposed and implemented into an elastic viscoplastic self-consistent (EVPSC) framework to simultaneously describe both stress and strain relaxation. The model captures the experimental data in terms of macroscopic stress strain curves, evolution of stress and strain during holding, as well as evolution of the internal elastic strains. Model results indicate that the magnitude of the stress relaxed during strain holding is dependent on both, the magnitude of the flow stress and the spread of the resolved shear stress distribution. The magnitude of strain accumulated during stress holding is, on the other hand, dependent on the magnitude of the hardening rate and on the spread of the resolved shear stress distribution. The internal elastic strains are directly correlated with the stress state, and hence the stress relaxation during strain holds has a greater influence on the lattice strains than strain relaxation during stress holds.
\end{abstract}

Keywords: Strain relaxation; Stress relaxation; Magnesium alloy; Neutron diffraction; Stress distribution.

\footnotetext{
* Corresponding author: Dr. Huamiao Wang, Email address: wanghm@lanl.gov. Tel.: 1-505-664-0321.
} 


\section{Introduction}

Magnesium alloys have been intensively investigated because they are light structural materials with a strong potential to reduce the weight of vehicles. While most research has focused on studying flow stress, anisotropy, ductility and failure, their creep properties have been investigated less (Spigarelli, 2008; Spigarelli and El Mehtedi, 2009). Interestingly, although stress and strain relaxations taking place at or in the vicinity of the flow stress at room temperature may be present during forming operations, such conditions have been investigated even less (MacEwen, 1982; Wang et al, 2013a). To understand such processes, in this work we gathered experimental information for developing a model of the visco-plastic mechanisms involved. We use in-situ neutron diffraction for simultaneously interrogating the flow response and the evolution of internal strains during monotonic loading, during loading with strain holds, and during loading with stress holds of rolled Mg AZ31 plate. To rationalize the experimental data, a novel dislocation-based relaxation model is introduced and implemented into an ElastoVisco-Plastic polycrystal formulation. The polycrystal model is then used to simulate the macroscopic relaxation experiments and, indirectly, benchmark our mesoscale model.

Resolving diffraction peaks with in-situ neutron diffraction to determine the lattice strains with a minimum accuracy of 100 microstrain $\left(\varepsilon=100 \times 10^{-6}\right)$ requires either to periodically interrupt the loading test while holding the strain or the stress constant for several minutes or, alternatively, to acquire data continuously while loading at very low strain rates $\left(\sim 10^{-5} \mathrm{~s}^{-1}\right)$. Stress relaxation is observed when holding strain, and strain relaxation is observed when holding stress. For example, Wang et al. (2013a) report a 10\% stress drop from the flow point when strainholding Stainless Steel in tension during 900 seconds at about 1.5 times the initial yield stress; and a strain increment of $\sim 1 \%$ when holding the stress constant at about 1.5 times the initial yield stress for about the same time. Since strain rates are not constant during holds, this complicates the interpretation of the experiment and the development of a constitutive law for the material. To overcome this shortcoming, an uninterrupted loading technique, which consists in collecting diffraction measurements continuously while the sample is loaded at a constant slow rate, has been applied to Stainless Steel (An et al., 2011; Wang et al. 2013a) and Zircaloy (Skippon and Daymond, 2015). The response corresponds to a monotonic strain rate, and the time interval used for integrating detector signals can be optimized to obtain desired diffraction peak accuracy. During the measurement the flow stress increases due to strain hardening. 
As a consequence, independently of the technique used, the challenge lies in explaining the experimental results in terms of a unique material model which accounts not only for monotonic loading, but also for combined stress or strain relaxation effects. As a matter of fact, the apparent handicap introduced by having to use stress or strain holds, is used in this work as a way to obtain experimental information for developing an elasto-visco-plastic model which both, accounts for and unravels, the relaxation mechanisms inside grains. Key in our model is to introduce stress fluctuations at the grain interior, and to consider the relaxation of these stress fluctuations via visco-plastic glide of dislocations. Such phenomena are missing in all of the previous modeling approaches described below.

Several models are available for describing the mechanical response of polycrystals: crystal-plasticity-based finite element (CP-FE) (Dawson et al, 2000; 2001; Bronkhorst et al, 2007; Abdolvand et al., 2011; Abdolvand and Daymond, 2012), crystal-plasticity-based Fast Fourier Transform (CP-FFT) models (Lebensohn et al., 2012) and mean-field effective-medium selfconsistent (SC) models (Lebensohn and Tomé, 1993; Wang et al., 2010c). While most of them are based on a visco-plastic response, in their present form none of them account for the physical mechanisms associated with relaxation. The CP-FE and CP-FFT models consider a single crystal or part of a single crystal as an element, and a visco-plastic law describes the constitutive response of slip systems at the integration points. These models account explicitly for grain morphology and interaction between a grain and its neighbors, and have been used to simulate neutron diffraction experiments (Dawson et al, 2000; 2001; 2005; Abdolvand et al., 2011; Abdolvand and Daymond, 2012; Kanjarla et al., 2012). In some of these works both the average peak shift and also its dispersion (peak width) were calculated (Dawson et al., 2005; Li and O’Dowd, 2011; Kanjarla et al., 2012). In agreement with experimental data, it is found that the peak width increases with increasing strain level, that is, with increased dislocation density. The measured distribution of the internal elastic strains (Balogh et al, 2012; Kanjarla et al, 2012) is directly connected to the distribution of the stress in the interior of the grains, and is our experimental window to the latter.

While CP-FE and CP-FFT models provide information about stress-strain intragranular inhomogeneity, they are far more computationally intensive than the effective medium selfconsistent polycrystal models. The latter treat the polycrystalline aggregate as a homogenous effective medium (HEM) with the average properties of the aggregate, and each grain as an 
ellipsoidal inclusion embedded in and interacting with the HEM. In this framework the inclusion is representative of all grains with the same orientation, and the HEM is an average representation of all possible neighbor arrangements. As a consequence, the assumption of HEM surrounding each grain is consistent with the intrinsic statistical character of the neutron diffraction measurement, and makes these polycrystal models well suited to interpret the measured internal elastic strains which are represented only by the average shift in the center of the peak position with respect to an unstrained reference value.

The elasto-plastic self-consistent (EPSC) model of (Turner and Tomé, 1994; Neil et al., 2010) has been extensively used in the past to interpret neutron diffraction data but it is not appropriate for the task at hand, since it is not based on a visco-plastic rate-sensitive law. Instead, in this work we adopt the elastic-visco-plastic self-consistent (EVPSC) model of (Molinari et al., 1997; Wang et al., 2010c) which has recently been used for interpreting in-situ neutron diffraction measurements because it is able to address monotonic and relaxation regimes using a single set of parameters (Wang et al., 2012a; 2013a).

Here we propose an added feature to enrich self-consistent models - that predict intergranular stresses but not the intra-granular stress distributions - via introducing stress fluctuations as a result of dislocation storage inside each deforming single crystal. Note that similar types of modifications would be necessary in full field methods too as they cannot resolve the internal stress fields arising from dislocations interactions. In a sense the approach proposed can be viewed as a computationally efficient alternative to micro-morphic models (Gurtin, 2000; Forest, 2009). We explore the added capabilities of this model in predicting stress or strain relaxation, and apply the model to simulate the complex neutron diffraction experiments described above.

\section{Experimental procedure}

The material used in the present work is a commercial AZ31 magnesium alloy having a nominal composition of $3 \mathrm{wt} . \% \mathrm{Al}$ and $1 \mathrm{wt} . \% \mathrm{Zn}$. The material came in the form of a $25.4 \mathrm{~mm}$ thick hot-rolled plate in the soft annealed condition (O temper) with an average grain size of 50 $\mu \mathrm{m}$. The flat dog-bone shaped tension samples $(89 \mathrm{~mm}$ long and had a $44 \mathrm{~mm}$ long rectangular gauge section with a height of $6.35 \mathrm{~mm}$ and a thickness of $5 \mathrm{~mm}$ ) and the cylindrical compression samples $(10 \mathrm{~mm}$ diameter and $24 \mathrm{~mm}$ long) were machined from the plate with the loading axis aligned with the transverse direction. The initial texture of the tested material was measured 
using the High Intensity Pressure and Preferred Orientation (HIPPO) instrument at Los Alamos Neutron Science Center (LANSCE) (Wenk et al., 2003). The initial texture used in the model simulations is derived from the experimental orientation distribution function and consists of a set of 14364 discrete orientations, by division of Euler space into $5^{\circ} \times 5^{\circ} \times 5^{\circ}$ cells, with volume fractions assigned to represent the measured initial texture. Fig. 1 shows the $\{00.1\},\{10.0\}$ and $\{10.1\}$ pole figures representing the discretized initial texture used for the simulations. The pole figures show that the as-received AZ31 plate had a very strong texture, evidenced by the strong $\{00.1\}$ intensity and absence of $\{10.0\}$ and $\{10.1\}$ intensity along the plate normal direction (ND). This texture is very typical of rolled magnesium alloy (Proust et al., 2009; Wu et al., 2010). In-situ neutron diffraction measurements are performed during compressive and tensile deformation using the Spectrometer for Materials Research at Temperature and Stress (SMARTS) at LANSCE. The specifics of SMARTS are described in detail elsewhere (Bourke et al., 2002), and here we only give a brief introduction. A servo-hydraulic load frame is oriented at a $45^{\circ}$ angle to the incident beam and thus the two detector banks at $\pm 90^{\circ}$ to the incident beam allows for simultaneous measurement of diffraction patterns with scattering vectors parallel and transverse to the loading axis, respectively. The time-of-flight technique allows for measuring full diffraction patterns in each detector bank. In all experiments the loading direction was along the transverse direction (TD) of the rolled plate. For the continuous loading measurements the neutron data are collected continuously throughout all the testing time using 5 minute count times, whereas for the stress- and strain-hold measurements the count time was nominally 20 minutes. Elastic lattice strains are calculated from changes in peak position in the diffraction patterns during deformation through the equation $\varepsilon^{h k \cdot l}=\left(d^{h k \cdot l}-d_{0}^{h k \cdot l}\right) / d_{0}^{h k . l}$. The stress-free reference lattice spacing for each peak, $d_{0}^{h k . l}$, was taken as the measured lattice spacing prior to applying any load to the sample. Hence, the $\{00.2\}$ reflection is omitted as the initial texture results in the absence of a $\{00.2\}$ peak, and thus we do not have a stress-free reference for it. This also means that we are neglecting any existing internal stresses stemming from the processing of the plate, but thermal intergranular stresses are not pronounced in magnesium alloys due to low thermal anisotropy. The experimental results will be presented in Section 4 .

Five tests of continuous tension, continuous compression, tension with stress holds, compression with stress holds and compression with strain holds have been performed. The macroscopic stress strain curves of these five tests are presented in Fig. 2a. The selected holding 
stress values for tensile and compressive tests are shown in Fig. $2 b$ and Fig. 2c, respectively. The selected holding strain values for the compression test are depicted in Fig. 2d. The holding times for the tests are also indicated in Figs. 2b, $2 c$ and 2 d. The holding times are not fully uniform, as some fluctuations in the incident neutron intensity occur due to source (accelerator) operations, resulting in different times required for obtaining the same counting statistic at each hold.

\section{Incorporating distributions in the constitutive model}

The EVPSC model expresses the shear rate contributed by the dislocations belonging to a particular slip system as a power of the resolved shear stress $\tau$ on that system

$$
\dot{\gamma}(\tau)=\dot{\gamma}_{0}\left|\frac{\tau}{\tau_{c}}\right|^{n} \operatorname{sgn}(\tau)
$$

where $\dot{\gamma}_{0}$ is reference shear rate with units of $s^{-1}$, and $\tau_{c}$ is a threshold stress. The power law introduces rate sensitivity: moving dislocations at a higher rate requires applying a higher resolved stress. In this work we significantly reduce such rate dependence by making $\dot{\gamma}_{0}$ proportional to the current macroscopic plastic strain rate (i.e., $\dot{\gamma}_{0}=\left|\overline{\dot{\boldsymbol{\varepsilon}}^{p}}\right|=\sqrt{\overline{\dot{\varepsilon}_{l \jmath}^{p}} \overline{\dot{\varepsilon}_{l \jmath}^{p}}}$, where $\overline{\dot{\boldsymbol{\varepsilon}}^{p}}$ is the average plastic strain rate over all the grains and, instead, introduce rate dependence through the constitutive law used to calculate $\tau_{c}$ (Beaudoin and Acharya, 2001; Beyerlein and Tomé, 2008).

Stress is not homogeneous in the interior of a grain, correspondingly, the resolved shear on a given slip system experiences fluctuations. In addition, dislocation segments will be activated at different threshold values with respect to the segment length and the local pinning mechanisms (Lagneborg et al., 1973). However, in effective medium models based on the ellipsoidal inclusion the stress is homogeneous inside each grain and there are no such fluctuations. As a consequence, the terms entering the shear rate given by Eq. (1) have to be interpreted as an effective resolved shear and an effective threshold shear for the grain. While the assumption of an effective threshold is justified when monotonic loading is involved, the fluctuations determine the rate of relaxation and have to be accounted for in the case of strain or stress holdings. Here we will: a) assume a Probability Distribution Function (PDF) for the fraction of dislocation segments in the grain experiencing a resolved shear stress $(\tau)$; b) the effect 
of distribution of dislocation segment is merged into the distribution of resolved shear stress (RSS); c) express the total shear rate contributed by a system in the grain as an integrated sum of the shear rates contributed by individual dislocations; (d) ignore any gradient in the distribution of resolved shear stress across the grain. The fact that diffraction peak shape is consistent with a Gaussian internal strain distribution (Li and O'Dowd, 2011; Kanjarla et al, 2012; Niezgoda et al., 2014) leads us to postulate a Gaussian distribution for the resolved shears, centered in the average RSS in the grain:

$$
p(\tau-\bar{\tau})=\frac{1}{\sqrt{2 \pi} V} \exp \left(-\frac{(\tau-\bar{\tau})^{2}}{2 V^{2}}\right)
$$

with $\int_{-\infty}^{\infty} p(\tau-\bar{\tau}) d \tau=1$. Here $\tau, \bar{\tau}$ and $V$ are the resolved shear stress (RSS), the average resolved shear stress (related to the macroscopic stress field through the effective medium localization law) and the standard deviation in the grain for a system. Figure 3 illustrates schematically the evolution of RSS distribution with hardening: as more dislocations are stored, the average CRSS increases, and so does the dispersion in the RSS distribution. While these distributions could be obtained from local FFT models and from discrete dislocation dynamic simulations the aim of this work is primarily to illustrate the role played by such distributions in the material behavior during stress or strain relaxation. As a consequence, here we adjust the standard deviation in Eq. (2) to experimental data.

With Eq. (2), we can define an effective shear rate for a slip/twinning system as the integral of shear rate over resolved shear stress weighted by the distribution function $p(\tau-\bar{\tau})$ :

$$
\dot{\gamma}_{e f f}=\int_{-\infty}^{\infty} \dot{\gamma}(\tau) p(\tau-\bar{\tau}) d \tau=\dot{\gamma}_{0}\left(\frac{\bar{\tau}}{\tau_{c}}\right)^{n}+\frac{n(n-1)}{4} \frac{(\sqrt{2} V)^{n}}{\tau_{c}^{n}} \dot{\gamma}_{0}\left(\frac{\bar{\tau}}{\sqrt{2} V}\right)^{n-2}+\cdots
$$

Details of the integration procedure and the derivation of Eq. (3) can be found in Appendix A.

The dislocation density hardening law is used to describe the hardening of the slip systems during deformation (Beyerlein and Tomé, 2008; Capolungo et al., 2009b). All slip systems are assigned the threshold stresses related to temperature and strain rate:

$$
\tau_{c}=\tau_{0}+\tau_{\text {for }}+\tau_{\text {sub }}
$$


The first component $\tau_{0}$ is the initial threshold stress, the second component $\tau_{\text {for }}$ is the contribution from a statistical distribution of stored dislocations $\rho_{t o t}=\sum_{\alpha} \rho^{\alpha}$. Its relationship to $\rho_{\text {tot }}$ is given by the Taylor relation:

$$
\tau=b \chi \mu \sqrt{\rho_{t o t}}
$$

where $\chi=0.2$ is the dislocation interaction parameter. The dislocation evolution is comprised of both storage and annihilation

$$
\frac{d \rho}{d \gamma}=k_{1} \sqrt{\rho}-k_{2} \rho
$$

where $k_{1}$ is a rate-insensitive coefficient for dislocation storage by statistical trapping of gliding dislocations by forest obstacles, and $k_{2}$ is the rate sensitive coefficient for dynamic recovery by thermally activated mechanisms, given by:

$$
\frac{k_{2}(\dot{\varepsilon}, T)}{k_{1}}=\frac{\chi b}{g}\left(1-\frac{k T}{D b^{3}} \ln \left(\frac{\dot{\varepsilon}}{\dot{\varepsilon}_{0}}\right)\right)
$$

where $k=1.38 \times 10^{-29}\left(\mathrm{MPa} \cdot \mathrm{m}^{3} / K\right), \dot{\varepsilon}_{0}, g$, and $D$ are Boltzman's constant, a reference strain rate $\left(\dot{\varepsilon}_{0}=10^{7} / \mathrm{s}\right)$, a normalized effective activation enthalpy, and a drag stress, respectively. As can be seen, the rate sensitivity of the material is accounted for in Eq. (7). The last term $\tau_{s u b}$ is the contribution from $\rho_{s u b}$, the stored dislocation density within organized substructure. An expression for that is provided by (Beyerlein and Tomé, 2008), based on an extended Taylor law (Basinski and Basinski, 1979; Madec et al., 2002)

$$
\tau_{\text {sub }}=k_{\text {sub }} \mu b \sqrt{\rho_{\text {sub }}} \log \left(\frac{1}{b \sqrt{\rho_{\text {sub }}}}\right)
$$

where $k_{\text {sub }}=0.086$ is a numerical constant, which ensures that the extended Taylor law converge to the usual Taylor law for small densities (Capolungo et al., 2009a). The substructure density $\rho_{\text {sub }}$ contains dislocations from all active glide dislocations in the grain, the rate of $\rho_{\text {sub }}$ development is coupled to the rate of recovery of all glide dislocations via:

$$
d \rho_{\text {sub }}=\sum_{\alpha} q^{\alpha}\left[k_{2}^{\alpha} \rho_{\text {for }}^{\alpha}\right] d \gamma^{\alpha}
$$

In the current study, we assume $\tau_{\text {sub }}^{\alpha}=0$ because there is not much debris at strain of $12 \%$.

The threshold stress for twinning assumed by the hardening model increases proportionally with the slip dislocation population:

$$
\tau_{c}^{\beta}=\tau_{0}^{\beta}+\tau_{\text {slip }}^{\beta}=\tau_{0}^{\beta}+\mu \sum_{\alpha} C^{\beta \alpha} b^{\beta} b^{\alpha} \rho^{\alpha}
$$


where $b^{\beta}$ is the magnitude of the Burgers vector for the twinning dislocation, and $C^{\beta \alpha}$ is the coefficient that suppress the growth of twinning system $\beta$ from the accumulation of the dislocations of slip system $\alpha$. Our predictions are rather insensitive to the precise values of $C^{\beta \alpha}$ used, indicating that the coupling term in Eq. 10 does not play a relevant role in the observed behavior.

The direct relation between the internal elastic strain and the RSS, suggests that the intragranular stress dispersion should increase with dislocation density, as does the dispersion of internal strain (Ungar et al., 1984; Balogh et al., 2012). As a consequence, the standard deviation $V^{\alpha}$ of the RSS on slip or twinning system $\alpha$ is assumed to be of the form

$$
V^{\alpha}=V=\eta \sqrt{\rho_{\text {tot }}}
$$

Here $\eta=10^{-7} \mathrm{MPa} / \mathrm{m}$ is a coefficient relating the standard deviation to the total dislocation density $\rho_{\text {tot }}=\sum_{\alpha=1}^{N_{\text {slip }}} \rho^{\alpha}$. Its value is adjusted so to obtain a realistic internal stress distribution. $V^{\alpha}$ represents the fluctuation in the resolved shear stress (RSS) that acts on slip/twinning system $\alpha$ in a grain. As a first step, we assume that it is the same for all systems because $V^{\alpha}$ originates from the total dislocation field in the grain. However, $V^{\alpha}$ is different from grain to grain because the total dislocation density varies from grain to grain.

The plastic strain rate of a single crystal is the sum of the shear strain rates on all slip/twinning systems $\left(\boldsymbol{b}^{\alpha}, \boldsymbol{n}^{\alpha}\right)$

$$
\dot{\varepsilon}_{i j}^{p}=\sum_{\alpha} \dot{\gamma}_{e f f}^{\alpha} m_{i j}^{\alpha}
$$

where $m_{i j}^{\alpha}=\left(b_{i}^{\alpha} n_{j}^{\alpha}+n_{i}^{\alpha} b_{j}^{\alpha}\right) / 2$ is the Schmid tensor. The average resolved shear stress is $\bar{\tau}^{\alpha}=\boldsymbol{\sigma}: \boldsymbol{m}^{\alpha}$ and the total plastic strain rate $\left(\dot{\varepsilon}_{i j}=\dot{\varepsilon}_{i j}^{e}+\dot{\varepsilon}_{i j}^{p}\right)$ can be linearized to

$$
\dot{\varepsilon}_{i j}=M_{i j k l}^{e} \dot{\sigma}_{k l}+M_{i j k l}^{p} \sigma_{k l}+\dot{\varepsilon}_{i j}^{0}
$$

where

$$
\dot{\varepsilon}_{i j}^{0}=\dot{\varepsilon}_{i j}^{p}-M_{i j k l}^{p} \sigma_{k l}
$$

and the viscoplastic compliance $M_{i j k l}^{p}$ is

$$
M_{i j k l}^{p}=\frac{\partial \dot{\varepsilon}_{i j}^{p}}{\partial \sigma_{k l}}=\sum_{\alpha} \frac{\partial \dot{\gamma}^{\alpha}}{\partial \bar{\tau}^{\alpha}} \frac{\partial \bar{\tau}^{\alpha}}{\partial \sigma_{k l}} m_{i j}^{\alpha}=\sum_{\alpha} \frac{\partial \dot{\gamma}^{\alpha}}{\partial \bar{\tau}^{\alpha}} m_{i j}^{\alpha} m_{k l}^{\alpha}
$$


where

$$
\frac{\partial \dot{\gamma}}{\partial \bar{\tau}}=n \dot{\gamma}_{0}\left(\frac{\bar{\tau}}{\tau_{c}}\right)^{n-1} \frac{1}{\tau_{c}}+\frac{n(n-1)(n-2)}{4 \sqrt{2} V} \frac{(\sqrt{2} V)^{n}}{\tau_{c}^{n}} \dot{\gamma}_{0}\left(\frac{\bar{\tau}}{\sqrt{2} V}\right)^{n-3}+\cdots
$$

The detailed derivation of Eq. (14b) can be found in Appendix A.

The foregoing constitutive model for a single crystal is implemented into the EVPSC framework for polycrystalline materials. A complete description of the EVPSC model can be found in Wang et al. (2010c; 2012b; 2013b; 2013c). The contribution of twinning to plastic deformation associated is calculated by the TDT model. TDT model introduces two operations associated with twinning, twin propagation and twin growth. Twin propagation creates a new twin across a twin free region of the grain, and splits the grain into an un-twinned orientation (matrix) and a twinned orientation (twin domain). Twin propagation is driven by the stress in the matrix. The growth of a twin is driven by both, the stress in the matrix (matrix reduction) and the stress in the twin (twin growth). Matrix reduction is active if the corresponding resolved shear stress in the matrix is positive, while twin growth is active only if the corresponding resolved shear stress in the twin is negative. Therefore TDT model enforces $\dot{\gamma} \neq 0$ only if the applied resolved shear stress is positive in matrix or negative in twins. A volume reduction in the matrix and a volume growth in the twin are accompanied. De-twinning is not accounted for because there is no loading path change to activate de-twinning in present study. For a detailed description of TDT model see Wang et al. (2012b; 2013b). Eq. (13) is the Affine linearization of the deformation in a single crystal, which was evaluated to give the best performance among various self-consistent linearization schemes (Lebensohn et al., 2007; Wang et al., 2010a; 2010b; 2010d).

\section{Results and discussions}

\subsection{Parameter determination}

Three types of slip systems are considered: basal $\langle a\rangle$ slip, Prismatic $\langle a\rangle$ slip and Pyramidal $\langle c+a\rangle$ slip, and one type of twinning system: $\{10.2\}$ extension twinning. The initial typical rolling texture of the AZ31 plate is shown in Fig. 1. The room temperature elastic constants of the single crystal are $C_{11}=58.0, C_{12}=25.0, C_{13}=20.8, C_{33}=61.2$ and $C_{44}=16.6 \mathrm{GPa}$ (Simmons and Wang, 1971). We calibrated model parameters through fitting the measured stress strain curves of AZ31 plate experimentally tested at $3 \times 10^{-6} \mathrm{~s}^{-1}$ under continuous tension and 
compression. Fig. 4 shows good agreement between the calculated curves from the statistical model and experimental curves for both monotonic tension and compression. The parameters calibrated against the experimental data are listed in Table 1 and used in all the following simulations involving strain and stress holds.

From these simulations, we can predict the relative activities of the slip and twinning systems. The results are shown in Fig. 5. We observe that the dominant deformation mechanism during tension is prismatic slip, while the dominant one during compression is twinning up to a strain of $6 \%$, after which twinning is exhausted, and the active mechanisms are basal, prismatic and pyramidal slip. The predicted twin volume fractions for compression are also reported in Fig. 5 and agree well with the experimental twinning volume fractions calculated from the integration of evolving texture components.

The predicted dislocation densities under tension and compression are of the order of $10^{14} \mathrm{~m}^{-2}$. Joni et al. (2013) have found that the dislocation density of a textured AZ31 magnesium alloy at $13 \%$ tensile deformation at room temperature was on the order of 1.8 to $3 \times 10^{14}$, which is consistent with our predictions. The value of the variance in the dislocation distribution $V$ is different from grain to grain because the total dislocation density varies from grain to grain. Fig. 6 shows that the average $V$ over all grains increases with deformation from 0 to around $1.5 \mathrm{MPa}$. Similar to the dislocation density, the average $V$ tends to saturate more rapidly with strain in the case of tension.

\subsection{Stress relaxation}

The distribution of the resolved shear stress (RSS) in the proposed statistical model plays an important role on stress relaxation (strain holding). As illustrated schematically in Fig. 7a, the average RSS $\bar{\tau}$ increases with increasing shear strain $\Gamma$. The RSS decreases during strain holding (A to C), while the CRSS remains nearly constant. Because of the power relation Eq. (1), the effective shear rate of a slip system arises mainly from those RSS's greater than the corresponding CRSS (shaded area in Figs. 7b-7d), even though it is integrated from $-\infty$ to $\infty$. Conceptually, the following picture is envisioned. During loading at a constant rate, the applied stress has to increase in order to overcome the ongoing hardening and activate the dislocation segments to accommodate the imposed deformation. In other words, the RSS $\bar{\tau}$ is always close to the CRSS $\tau_{c}$, the shaded area is almost constant, and the effect on mechanical response from the 
stress distribution is less prominent. During strain holding, the decrease of the average RSS shifts the RSS distribution to the left of the CRSS and results in a decrease of the active dislocation segments (smaller shaded area in Figs $7 \mathrm{c}$ and $7 \mathrm{~d}$ ). Therefore the effective shear rate of the slip system decreases as the holding time increases.

Experimentally, an in-situ compressive test along TD was conducted with strain holdings at pre-determined strains, during which time neutron diffraction data were collected for the duration of the hold. For the simulation, the loading and reload strain rates are prescribed to be the same as in the experiment, i.e. $10^{-4} s^{-1}$. The boundary conditions for the strain holding are: $\dot{\varepsilon}_{11}=0$ and $\sigma_{i j}=0$ except for $\sigma_{11}$. The holding times at each strain are prescribed to be equal to the experimental ones (reported in Fig. 2d), which is required in order to make a fair comparison of the measured and predicted stress drop. As relaxation takes place the RSS on every system decreases, and so does the plastic strain rate $\dot{\boldsymbol{\varepsilon}}^{p}$. Computationally, the reference shear rate $\dot{\gamma}_{0}=\left|\overline{\dot{\boldsymbol{\varepsilon}}^{p}}\right|$ is adjusted in Eq. 3, which removes the rate effect induced by the power $n$ (in practice, the $\dot{\boldsymbol{\varepsilon}}^{p}$ of the previous increment is used to calculate $\dot{\gamma}_{0}$ ). Therefore the rate effect comes mostly from the dependence of $\tau_{c}$ with strain rate, which is small.

The measured and simulated stress strain curves under compression with strain holdings are compared in Fig. 8. Good agreement is obtained for the combined successions of loading and strain holding up to the strain level of $6 \%$. Beyond that level the stresses relaxed are slightly under-predicted by the model. Perhaps more important is the detailed evolution of the stress during the holding time. Fig. 9 compares the measured and predicted evolutions of the applied stress $\sigma$ with time during strain holdings under both compression and tension (only numerical results are available for tension). The stress drops rapidly at the beginning of holding and then decays at longer times. The predicted stress drops are generally in good agreement with experimental ones.

We expect that stress relaxation will depend strongly on the individual contribution of the slip and twinning mechanisms involved. We find that systems contribute very differently during monotonic loading than during strain holds. Fig. 10 depicts relative activity during load, holds and reloads for the compression test. While the relative activity tends to be the same during the reload portions as in the monotonic loading case (Fig 5a), it changes considerably during the holds. Basal shear-rate increases and twinning shear-rate decreases, although twinning is still activated and grow during holds. Fig. 10a presents the predicted relative activities of deformation 
mechanisms under compression with strain holds as a function of strain (mainly during loading). Fig. 10b shows the same activities as a function of time (mainly during holdings). Observe that twin volume fraction only increases significantly during the reload stages, and mostly at small strains (time $<16000$ s or $\varepsilon<7 \%$ ). Basal slip and prismatic slip are active afterwards (time $>16000$ s or $\varepsilon>7 \%$ ). During holding, the relative activities of the deformation mechanisms with lower CRSSs decrease, while the relative activities of systems with higher CRSS's increase (Fig. 10c). This occurs because the same absolute decrease in RSS results in a larger relative shift 'to the left' of the RSS distribution in Fig. 7, so less dislocation segments experiencing a RSS $>$ CRSS. Furthermore, in both the experiment and simulation, the increasingly larger drop in stress at larger plastic strains present can be explained in terms of increasing dislocation density: Because the spread $V$ of the RSS distribution increases with dislocation density (Eq. 11), more dislocation segments experience a RSS $>$ CRSS when the stress is relaxed (Fig. 7), and so they contribute more shear during the hold. The monotonic increase of $V$ responsible for the increase of the relaxed stress is reported in Fig. 6.

The distribution of the total dislocation density among grains at the start and end of stress relaxation for the compression test with strain holds is depicted in Fig. 11 at strains of $6 \%$ and $10 \%$. The frequency $f$ is computed by adding the total weight of grains with dislocation density falling in 20 consecutive intervals in the range from 0 to $6 \times 10^{14} \mathrm{~m}^{-2}$. Fig. 11 clearly shows the dispersion in dislocation density (i.e. hardening) from grain to grain, and that the dislocation density increases with straining. The comparison between the frequencies of dislocation density at the start and end of the relaxation process shows no significant evolution during stress relaxation. This is reasonable because the drop of stress during holding has associated very little plastic deformation.

\subsection{Strain relaxation}

During stress holding the RSS on slip and twin systems remains constant but, because dislocations are being stored their density increases and so does the CRSS. The situation is illustrated in Fig. 12a, (A to C): the shift 'to the right' of the CRSS means that less segments have shear rates that contribute substantially to shear (smaller shaded area in Figs 12c and 12d), and the integrated effective shear rate (Eq. 3) decreases. 
The uniaxial deformation with stress holds is simulated here using the same constitutive model and parameters, but with boundary conditions of zero macroscopic stress rate $\dot{\sigma}_{i j}=0$. The holding times imposed are equal to the experimental times shown in Figs. 2b and 2c. In Fig. 13, the predicted stress strain curves are compared with the experimental ones. Notably, the predictions of total strain relaxation during each hold agree well qualitatively and quantitatively with the experiments, which supports the validity of the visco-plastic stress fluctuation model proposed here.

Observe that the experimental data is reported in engineering stress-strain values. In order to compare with model predictions given in true stress-strain values, it is necessary to convert one to the other. For uniaxial loading, the relation between the engineering and true components is given by $\sigma^{\text {true }}=\sigma^{e n g}\left(1+\varepsilon^{e n g}\right)$. During strain holdings the length of the sample remains invariant, and the conversion above from engineering to true stress and strain is straightforward. The boundary condition $\dot{\varepsilon}_{i j}^{t o t a l}=0$ applied for strain holding is the same for true or engineering strain, and consistent with the experimental one. However, for the case of stress hold the relaxation strains are a few \% (Fig 13a) and a constant engineering stress does not ensure a constant true stress because the section of the sample changes during the hold. As a consequence, the stress-hold simulation condition of constant true stress is not consistent with the experimental condition of constant engineering stress. Fig. 13b depicts the engineering and true stress strain curves under tension and compression with stress holds. While for the comparison we can (and do) correct true strain to engineering strain, a constant engineering stress results in an increasing true stress for tensile holds and a decreasing true stress for compression holds (Fig. 13b). The simulated true stress rate is zero but the experimental true stress rate is $\dot{\sigma}^{\text {true }}=\sigma^{\text {eng }} \dot{\varepsilon}^{\text {eng }}$. Because the magnitude of $\dot{\varepsilon}^{e n g}$ decreases to nearly zero during holds, $\dot{\sigma}^{\text {true }}$ is small and the difference between the experimental and numerical boundary conditions will affect only slightly the final results.

Fig. 14 shows the detailed evolution of the strain during holding time. We observe that the strain accumulates with time during stress holding at a decreasing rate. Good agreement between the predicted and experimental evolutions of the strain during holdings is obtained for compression. For tension, good agreement is also obtained at the lower stress levels, while at higher stress (e.g., $240 \mathrm{MPa}$ ) levels the model slightly under predicts relaxation. 
The discrepancy can be connected with the hardening rate, which is high at low plastic strains and low at high plastic strains. Specifically, the amount of strain accommodated during stress holding is dependent on the evolution of flow stress (i.e., CRSSs of active systems) during holding, which in turn is dependent on the hardening rate $(d \sigma / d \varepsilon)$. A higher hardening rate results in a faster increase of the flow stress with strain, and therefore an earlier arrest of dislocations and smaller accumulated strain during stress holding. This behavior is consistent with both the predicted and experimental data shown in Fig. 14. When the hardening rate is high, a small strain is accumulated during holding (e.g., holding at stress of -190 MPa under compression). In contrast, when the hardening rate is low, large strain is accumulated during stress holding (e.g., holding at stress of $240 \mathrm{MPa}$ under tension). Last, we add that the monotonic increase of $V$ (see Fig. 6) also results in the increase of the strain accumulated during stress holding. The system activity for strain relaxation shows no significant difference from the continuous one and therefore is not reported.

\subsection{Internal elastic strain}

A valuable result from the in-situ neutron diffraction measurement is the evolution of internal lattice strains. This experimental data comes from subsets of grains, and so provides a stringent test of the model calculations and insight into local behavior. Fig. 15a compares the measured and predicted lattice strains of $\{11.0\}$ and $\{10.1\}$ diffraction planes under continuous compression. The model successfully captures the trend of the lattice strains measured by neutron diffraction, up to a load of $200 \mathrm{MPa}$, when most of twinning reorientation has already taken place (Fig 5a). Results tend to diverge past such point, possibly because the slip activity in the twinned domains causes a load transfer that is not properly captured by the model. In addition, twinning is also responsible for the dispersion observed for $\{11.0\}$ peaks above $300 \mathrm{MPa}$, because twinning reorients the grains that contribute to the peak and only a small volume fraction remains, rendering the measured lattice strains noisy. We were not able to assess the lattice strains for the $\{00.2\}$ reflection as the initial texture caused an absence of the $\{00.2\}$ peak in the loading direction. Consequently, we do not have a directly measured stress-free lattice parameter for this reflection.

The effect of holdings on the evolution of the lattice strains $(\{11.0\}$ and $\{10.1\})$ under compression is presented in Figs. $15 \mathrm{~b}$ and $15 \mathrm{c}$. The main curve follows the behavior of the 
continuous load case, except that each stress hold leads to strain relaxation and a horizontal shift in the micro-strain response. In the case of macroscopic strain holds both, the stress and the elastic strain on specific crystallographic planes are relaxed, and it is obvious from Fig. 15c that strain holding affects the evolution of lattice strain more significantly than stress holding. To complement the information discussed above, we present the evolution of the $\{11.0\}$ lattice strain for a few holding instances in Fig. 16. During stress holding, the lattice strain stays nearly constant, except for a slight decrease with time. During strain holding, the lattice strain decreases rapidly at the beginning and stabilizes as the holding time increases. The lattice strain decreases more at higher strain level (or stress level).

\section{Conclusions}

Stress and strain relaxation in magnesium alloy AZ31 plate are investigated both experimentally and numerically. The in-situ neutron diffraction technique is used to measure the macroscopic stress-strain response and internal elastic strain of AZ31 plate during deformation. In parallel, a constitutive model is proposed to predict the stress and strain relaxation during hold times, which postulates a Resolved Shear Stress (RSS) distribution acting on each slip or twinning plane. The dispersion of the distribution increases with increasing dislocation density. Such feature, combined with a visco-plastic activation law for dislocations, is implemented into an Elasto-Visco-Plastic polycrystal (EVPSC) framework and is applied to simulate the mechanical behavior of polycrystalline Mg AZ31 plate.

In a previous similar study of Stainless Steel (Wang et al, 2013a) we were able to reproduce the stress and strain relaxation associated with strain and stress holds, respectively, by using a more empirical approach. Specifically: an empirical Voce law for slip system hardening and a relatively rate insensitive response of slip systems $(n=1 / m=60)$. Such approach was not successful for describing the variation of the stress relaxation or strain relaxation intervals as the total test strain increases. Instead, the drops in stress or the creep strains predicted tended to be rather constant, and independent of the point in the flow curve at which the hold is imposed. Recent simulations of holds in Zircaloy done by Skippon and Daymond (2015) (similar as those done by Wang et al (2013a) for Stainless Steel) show the same behavior and miss the variable amount of relaxation. It was because of these results that we decided to adopt the dislocation based statistical approach to describe relaxation presented here. With the increase of the variable 
standard deviation $V$ with straining, the model correctly captures the variable amount of relaxation. Such approach should also work for other materials. This work shows that when several slip and twin systems are present, each with different threshold activation stress, they contribute in different amounts and at different rates to relaxation. Such situation cannot be handled with rate independent empirical hardening laws, and a single rate sensitivity parameter.

The model presented here applies to a less studied regime, namely, stress and strain relaxation under conditions close to or at the flow stress of the material and at a relatively low homologous temperature. We avoided referring to this regime as 'creep' because this is used in connection with lower-than-flow stress, and high temperature. We foresee that, in addition to the relaxation regime, our statistical model will help explain mechanical response associated with strain rate changes used in experiments designed to characterize rate sensitivity.

Other conclusions of this work, discussed in Section 4, are:

1. The behavior of the AZ31 plate under strain or stress holding is consistent, qualitatively and quantitatively, with fluctuations in the RSS acting on slip/twin systems described by a Gaussian distribution, with a variance proportional to the dislocation density. Future simulations with Discrete Dislocation Dynamics will help us decide the precise characteristics of such distribution.

2. During strain holding, the average RSS in the grain decreases and shifts the distribution away from the threshold stress, which reduces the fraction of dislocation segments being activated. Therefore, the rapid stress relaxation at the beginning of the hold slows down considerably with time. During stress holding, on the other hand, the RSS stays constant but the threshold increases due to dislocation storage and associated hardening. Therefore the strain initially accumulates quickly, but its rate gradually decreases with holding time.

3. In addition to the shift of the RSS distribution, the stress and strain relaxation are also dependent on the broadness of the RSS distribution. Broader distributions result in larger relaxed stress during strain holding and larger accumulated strain during stress holding.

4. The amount of relaxed stress during strain holding depends on the CRSS of the system. Active systems with a high CRSS are more effective in relaxing stress. In the 
case of stress holding, on the other hand, relaxation depends on the hardening rate of the active systems. A higher hardening rate results in smaller accumulated strain during stress holding.

\section{Acknowledgement}

This work is fully funded by the U.S. Dept. of Energy, Office of Basic Energy Sciences Project FWP 06SCPE401. This work has benefited from the use of SMARTS and HIPPO at the Lujan Center at Los Alamos Science Center. Los Alamos National Laboratory is operated by Los Alamos National Security LLC under DOE contract DE-AC52-06NA25396. 


\section{Appendix A}

To get the effective shear rate on a given system (Eq. (3)), it is necessary to calculate the following integral,

$$
\int_{-\infty}^{\infty}\left(\frac{\tau}{\tau_{c}}\right)^{n} p(\tau-\bar{\tau}) d \tau=\frac{1}{\sqrt{2 \pi} V} \int_{-\infty}^{\infty}\left(\frac{\tau}{\tau_{c}}\right)^{n} e^{-\frac{(\tau-\bar{\tau})^{2}}{2 V^{2}}} d \tau \stackrel{x=\frac{\tau-\bar{\tau}}{\sqrt{2} V}}{\Longrightarrow} \frac{(\sqrt{2} V)^{n}}{\sqrt{\pi} \tau_{c}^{n}} \int_{-\infty}^{\infty}\left(x+\frac{\bar{\tau}}{\sqrt{2} V}\right)^{n} e^{-x^{2}} d x
$$

From the binomial theorem,

$$
\left(x+\frac{\bar{\tau}}{\sqrt{2} V}\right)^{n}=\sum_{k=0}^{n}\left(\begin{array}{l}
n \\
k
\end{array}\right) x^{k}\left(\frac{\bar{\tau}}{\sqrt{2} V}\right)^{n-k}=\sum_{k=0}^{n}\left(\frac{\bar{\tau}}{\sqrt{2} V}\right)^{n-k}\left(\begin{array}{l}
n \\
k
\end{array}\right) x^{k}
$$

with

$$
\left(\begin{array}{l}
n \\
k
\end{array}\right)=\frac{n !}{k !(n-k) !}
$$

therefore

$$
\int_{-\infty}^{\infty}\left(\frac{\tau}{\tau_{c}}\right)^{n} p(\tau-\bar{\tau}) d \tau=\frac{(\sqrt{2} V)^{n}}{\sqrt{\pi} \tau_{c}^{n}}\left(\sum_{k=0}^{n}\left(\frac{\bar{\tau}}{\sqrt{2} V}\right)^{n-k}\left(\begin{array}{l}
n \\
k
\end{array}\right) \int_{-\infty}^{\infty} x^{k} e^{-x^{2}} d x\right)
$$

The following integral is useful

$$
\begin{aligned}
\int x^{k} e^{-x^{2}} d x=\frac{1}{2} \int x^{k-1} e^{-x^{2}} d x^{2}=-\frac{1}{2} \int x^{k-1} d e^{-x^{2}}=-\frac{1}{2} x^{k-1} e^{-x^{2}}+\frac{(k-1)}{2} \int x^{k-2} e^{-x^{2}} d x \\
=-\frac{1}{2} x^{k-1} e^{-x^{2}}-\frac{(k-1)}{4} x^{k-3} e^{-x^{2}}-\frac{(k-1)(k-3)}{8} x^{k-5} e^{-x^{2}} \\
-\frac{(k-1)(k-3)(k-5)}{16} x^{k-7} e^{-x^{2}}+\cdots+T_{\text {last }}
\end{aligned}
$$

If $k$ is an odd number, $\int x^{k} e^{-x^{2}} d x=0$. If $k$ is an even number, the last term will be

$$
\begin{gathered}
T_{\text {last }}=\int e^{-x^{2}} d x=\frac{\sqrt{\pi}}{2} \operatorname{erf}(x)+C \\
\int_{-\infty}^{\infty} x^{k} e^{-x^{2}} d x=\left.\sum_{i=1}^{\frac{k}{2}-1} C_{i} x^{k-2 i+1} e^{-x^{2}}\right|_{-\infty} ^{\infty}+\left.\frac{\prod_{i=1}^{\frac{k}{2}}(k-2 i+1)}{2^{\frac{k}{2}}} \frac{\sqrt{\pi}}{2} \operatorname{erf}(x)\right|_{-\infty} ^{\infty}=\frac{\prod_{i=1}^{\frac{k}{2}}(k-2 i+1)}{2^{\frac{k}{2}}} \sqrt{\pi}
\end{gathered}
$$

So if $k$ is an even number $(k>2)$

$$
\int_{-\infty}^{\infty} x^{k} e^{-x^{2}} d x=\left.\frac{\prod_{i=1}^{\frac{k}{2}}(k-2 i+1)}{2^{\frac{k}{2}}} \frac{\sqrt{\pi}}{2} \operatorname{erf}(x)\right|_{-\infty} ^{\infty}=\frac{\prod_{i=1}^{\frac{k}{2}}(k-2 i+1)}{2^{\frac{k}{2}}} \sqrt{\pi}
$$

Since $\int_{-\infty}^{\infty} x^{k} e^{-x^{2}} d x$ is zero if $\boldsymbol{k}$ is an odd number,

$$
\frac{(\sqrt{2} V)^{n}}{\sqrt{\pi} \tau_{c}^{n}}\left(\sum_{k=0,2,4 \ldots}^{n}\left(\frac{\bar{\tau}}{\sqrt{2} V}\right)^{n-k}\left(\begin{array}{l}
n \\
k
\end{array}\right) \int_{-\infty}^{\infty} x^{k} e^{-x^{2}} d x\right)=T_{0}+T_{2}+T_{4}+\cdots T_{n}\left(\text { or } T_{n-1}\right)
$$




$$
\begin{aligned}
& T_{0}=\frac{(\sqrt{2} V)^{n}}{\sqrt{\pi} \tau_{c}^{n}}\left(\frac{\bar{\tau}}{\sqrt{2} V}\right)^{n} \sqrt{\pi}=\frac{(\sqrt{2} V)^{n}}{\tau_{c}^{n}}\left(\frac{\bar{\tau}}{\sqrt{2} V}\right)^{n}=\left(\frac{\bar{\tau}}{\tau_{c}}\right)^{n} \\
& T_{2}(k=2)=\frac{(\sqrt{2} V)^{n}}{\sqrt{\pi} \tau_{c}^{n}}\left(\frac{\bar{\tau}}{\sqrt{2} V}\right)^{n-2}\left(\begin{array}{l}
n \\
2
\end{array}\right) \int_{-\infty}^{\infty} x^{2} e^{-x^{2}} d x=\frac{(\sqrt{2} V)^{n}}{\sqrt{\pi} \tau_{c}^{n}}\left(\frac{\bar{\tau}}{\sqrt{2} V}\right)^{n-2} \frac{n(n-1)}{2} \frac{(k-1)}{2} \int_{-\infty}^{\infty} e^{-x^{2}} d x \\
& =\frac{n(n-1)}{4} \frac{(\sqrt{2} V)^{n}}{\tau_{c}^{n}}\left(\frac{\bar{\tau}}{\sqrt{2} V}\right)^{n-2} \\
& T_{4}(k=4)=\frac{(\sqrt{2} V)^{n}}{\sqrt{\pi} \tau_{c}^{n}}\left(\frac{\bar{\tau}}{\sqrt{2} V}\right)^{n-4}\left(\begin{array}{c}
n \\
4
\end{array}\right) \int_{-\infty}^{\infty} x^{4} e^{-x^{2}} d x \\
& =\frac{(\sqrt{2} V)^{n}}{\sqrt{\pi} \tau_{c}^{n}}\left(\frac{\bar{\tau}}{\sqrt{2} V}\right)^{n-4} \frac{n(n-1)(n-2)(n-3)}{24} \frac{(k-1)(k-3)}{4} \int_{-\infty}^{\infty} e^{-x^{2}} d x \\
& =\frac{n(n-1)(n-2)(n-3)}{32} \frac{(\sqrt{2} V)^{n}}{\tau_{c}^{n}}\left(\frac{\bar{\tau}}{\sqrt{2} V}\right)^{n-4} \\
& T_{6}(k=6)=\frac{(\sqrt{2} V)^{n}}{\sqrt{\pi} \tau_{c}^{n}}\left(\frac{\bar{\tau}}{\sqrt{2} V}\right)^{n-6}\left(\begin{array}{c}
n \\
6
\end{array}\right) \int_{-\infty}^{\infty} x^{6} e^{-x^{2}} d x \\
& =\frac{(\sqrt{2} V)^{n}}{\sqrt{\pi} \tau_{c}^{n}}\left(\frac{\bar{\tau}}{\sqrt{2} V}\right)^{n-6} \frac{n(n-1) \ldots(n-4)(n-5)}{720} \frac{(k-1)(k-3)(k-5)}{8} \int_{-\infty}^{\infty} e^{-x^{2}} d x \\
& =\frac{n(n-1) \ldots(n-4)(n-5)}{384} \frac{(\sqrt{2} V)^{n}}{\tau_{c}^{n}}\left(\frac{\bar{\tau}}{\sqrt{2} V}\right)^{n-6} \\
& T_{8}(k=8)=\frac{(\sqrt{2} V)^{n}}{\sqrt{\pi} \tau_{c}^{n}}\left(\frac{\bar{\tau}}{\sqrt{2} V}\right)^{n-8}\left(\begin{array}{l}
n \\
8
\end{array}\right) \int_{-\infty}^{\infty} x^{8} e^{-x^{2}} d x \\
& =\frac{(\sqrt{2} V)^{n}}{\sqrt{\pi} \tau_{c}^{n}}\left(\frac{\bar{\tau}}{\sqrt{2} V}\right)^{n-8} \frac{n(n-1) \ldots(n-6)(n-7)}{8 !} \frac{(k-1)(k-3)(k-5)(k-7)}{16} \int_{-\infty}^{\infty} e^{-x^{2}} d x \\
& =\frac{n(n-1) \ldots(n-6)(n-7)}{6144} \frac{(\sqrt{2} V)^{n}}{\tau_{c}^{n}}\left(\frac{\bar{\tau}}{\sqrt{2} V}\right)^{n-8} \\
& T_{10}(k=10)=\frac{(\sqrt{2} V)^{n}}{\sqrt{\pi} \tau_{c}^{n}}\left(\frac{\bar{\tau}}{\sqrt{2} V}\right)^{n-10}\left(\begin{array}{c}
n \\
10
\end{array}\right) \int_{-\infty}^{\infty} x^{10} e^{-x^{2}} d x \\
& =\frac{(\sqrt{2} V)^{n}}{\sqrt{\pi} \tau_{c}^{n}}\left(\frac{\bar{\tau}}{\sqrt{2} V}\right)^{n-10} \frac{n(n-1) \ldots(n-8)(n-9)}{10 !} \frac{(k-1)(k-3)(k-5)(k-7)(k-9)}{32} \int_{-\infty}^{\infty} e^{-x^{2}} d x \\
& =\frac{n(n-1) \ldots(n-8)(n-9)}{122880} \frac{(\sqrt{2} V)^{n}}{\tau_{c}^{n}}\left(\frac{\bar{\tau}}{\sqrt{2} V}\right)^{n-8}
\end{aligned}
$$

$$
\int_{-\infty}^{\infty}\left(\frac{\tau}{\tau_{c}}\right)^{n} p(\tau-\bar{\tau}) d \tau=T_{0}+T_{2}+T_{4}+\cdots
$$

The derivative of $\int_{-\infty}^{\infty}\left(\frac{\tau}{\tau_{c}}\right)^{n} p(\tau-\bar{\tau}) d \tau$ is therefore the sum of the following terms

$$
\frac{\partial T_{0}}{\partial \bar{\tau}}=n\left(\frac{\bar{\tau}}{\tau_{c}}\right)^{n-1} \frac{1}{\tau_{c}}
$$




$$
\begin{gathered}
\frac{\partial T_{2}}{\partial \bar{\tau}}=\frac{n(n-1)(n-2)}{4 \sqrt{2} V} \frac{(\sqrt{2} V)^{n}}{\tau_{c}^{n}}\left(\frac{\bar{\tau}}{\sqrt{2} V}\right)^{n-3} \\
\frac{\partial T_{4}}{\partial \bar{\tau}}=\frac{n(n-1)(n-2)(n-3)(n-4)}{32 \sqrt{2} V} \frac{(\sqrt{2} V)^{n}}{\tau_{c}^{n}}\left(\frac{\bar{\tau}}{\sqrt{2} V}\right)^{n-5} \\
\frac{\partial T_{6}}{\partial \bar{\tau}}=\frac{n(n-1) \ldots(n-5)(n-6)}{384 \sqrt{2} V} \frac{(\sqrt{2} V)^{n}}{\tau_{c}^{n}}\left(\frac{\bar{\tau}}{\sqrt{2} V}\right)^{n-7} \\
\frac{\partial T_{8}}{\partial \bar{\tau}}=\frac{n(n-1) \ldots(n-7)(n-8)}{6144 \sqrt{2} V} \frac{(\sqrt{2} V)^{n}}{\tau_{c}^{n}}\left(\frac{\bar{\tau}}{\sqrt{2} V}\right)^{n-9} \\
\frac{\partial T_{10}}{\partial \bar{\tau}}=\frac{n(n-1) \ldots(n-10)}{122880 \sqrt{2} V} \frac{(\sqrt{2} V)^{n}}{\tau_{c}^{n}}\left(\frac{\bar{\tau}}{\sqrt{2} V}\right)^{n-11} \\
\text { i.e., } \quad \frac{\partial \int_{-\infty}^{\infty}\left(\frac{\tau}{\tau_{c}}\right)^{n} p(\tau-\bar{\tau}) d \tau}{\partial \bar{\tau}}=\frac{\partial T_{0}}{\partial \bar{\tau}}+\frac{\partial T_{2}}{\partial \bar{\tau}}+\cdots
\end{gathered}
$$




\section{References}

Abdolvand H.; Daymond M.R., Mareau C., 2011. Incorporation of twinning into a crystal plasticity finite element model: Evolution of lattice strains and texture in Zircaloy-2. International Journal of Plasticity 27, 1721-1738.

Abdolvand H.; Daymond M.R., 2012. Internal strain and texture development during twinning: Comparing neutron diffraction measurements with crystal plasticity finite-element approaches. Acta Materialia 60, 2240-2248.

An K., Skorpenske H.D., Stoica A.D., 2011. First in situ lattice strains measurements under load at VULCAN. Metallurgical and Materials Transactions A 42 95-99.

Balogh L., Capolungo L., Tomé C.N., 2012. On the measure of dislocation densities from diffraction line profiles: A comparison with discrete dislocation methods. Acta Materialia 60, 1467-1477.

Basinski S.J., Basinski Z.S., 1979. Dislocation in solids, in: Nabarro, F.R.N. (Ed.), North Holland, Amsterdam.

Beaudoin A.J., Acharya A., 2001. A model for rate-dependent flow of metal polycrystals based on the slip plane lattice incompatibility. Materials Science and Engineering A309-310, 411-415.

Beyerlein I.J., Tomé C.N., 2008. A dislocation-based constitutive law for pure Zr including temperature effects. International Journal of Plasticity 24, 867-895.

Bourke M.A.M., Dunand D.C., Ustundag E., 2002. SMARTS - a spectrometer for strain measurement in engineering materials. Applied Physics A 74 [Suppl.], S1707-S1709.

Bronkhorst C.A., Hansen B.L., Cerreta E.K., Bingert J.F., 2007. Modeling the microstructural evolution of metallic polycrystalline materials under localization conditions. Journal of the Mechanics and Physics of Solids 55, 2351-2383

Capolungo L., Beyerlein I.J., Tomé C.N., 2009a. Slip-assisted twin growth in hexagonal closepacked metals. Scripta Materialia 60, 32-35.

Capolungo L., Marshall P., McCabe R.J., Beyerlein I.J., Tomé C.N., 2009b. Nucleation and growth of twins in Zr: A statistical study. Acta Materialia 57, 6047-6056.

Dawson P.R., Boyce D.E., MacEwen S.R., Rogge R.B., 2000. Residual strains in HY100 polycrystals: Comparisons of experiments and simulations. Metallurgical and Materials Transactions A 31, 1543-1555.

Dawson P.R., Boyce D.E., MacEwen S.R., Rogge R.B., 2001. On the influence of crystal elastic moduli on computed lattice strains in AA-5182 following plastic straining. Materials Science and Engineering A313, 123-144.

Dawson P.R., Boyce D.E., Rogge R.B., 2005. Correlation of diffraction peak broadening to crystal strengthening in finite element simulations. Materials Science and Engineering A399, 13-25.

Forest S., 2009. Micromorphic Approach for gradient elasticity, viscoplasticity, and damage. Journal of Engineering Mechanics-ASCE 135, 117-131.

Gurtin M.E., 2000. On the plasticity of single crystals: Free energy, microforce, plastic-strain gradient. Journal of the Mechanics and Physics of Solids 48, 989-1036.

Joni B., Al-Samman T., Chowdhury S.G., Csiszar G., Ungar T., 2013. Dislocation densities and prevailing slip-system types determined by X-ray line profile analysis in a textured AZ31 magnesium alloy deformed at different temperatures. Journal of Applied Crystallography $46,55-62$. 
Kanjarla A.K., Lebensohn R.A., Balogh L., Tomé C.N., 2012. Study of internal lattice strain distributions in stainless steel using a full-field elasto-viscoplastic formulation based on fast Fourier transforms. Acta Materialia 60, 3094-3106.

Lagneborg R., Forsen B.H., 1973. A model based on dislocation distributions for workhardening and the density of mobile and immobile dislocations during plastic flow. Acta Metallurgica 21, 781-790.

Lebensohn R.A., Kanjarla A.K., Eisenlohr P., 2012. An elasto-viscoplastic formulation based on fast Fourier transforms for the prediction of micromechanical fields in polycrystalline materials. International Journal of Plasticity 32-33, 59-69.

Lebensohn R.A., Tomé C.N., 1993. A self-consistent anisotropic approach for the simulation of plastic deformation and texture development of polycrystals: application to zirconium alloys. Acta Metallurgica et Materialia 41, 2611-2624.

Lebensohn R.A., Tomé C.N., Ponte Castañeda P., 2007. Self-consistent modeling of the mechanical behavior of viscoplastic polycrystals incorporating intragranular field fluctuations. Philosophical Magazine 87, 4287-4322.

Li D.F., O'Dowd N.P., 2011. On the evolution of lattice deformation in austenitic stainless steels-The role of work hardening at finite strains. Journal of the Mechanics and Physics of Solids 59, 2421-2441.

MacEwen S.R., 1982. The mechanical equation of state and strengthening mechanisms for deformation of crystal-bar zirconium. Acta Metallurgica 30, 1431-1442.

Madec R., Devincre B., Kubin L.P., 2002. From dislocation junctions to forest hardening. Physical Review Letters 89.

Molinari A., Ahzi S., Kouddane R., 1997. On the self-consistent modeling of elastic-plastic behavior of polycrystals. Mechanics of Materials 26, 43-62.

Neil C.J., Wollmershauser J.A., Clausen B., Tomé C.N., Agnew S.R., 2010. Modeling lattice strain evolution at finite strains and experimental verification for copper and stainless steel using in situ neutron diffraction. International Journal of Plasticity 26, 1772-1791.

Niezgoda S.R., Kanjarla A.K., Beyerlein I.J., Tomé C.N., 2014. Stochastic modeling of twin nucleation in polycrystals: An application in hexagonal close-packed metals. International Journal of Plasticity 56, 119-138.

Proust G., Tomé C.N., Jain A., Agnew S.R., 2009. Modeling the effect of twinning and detwinning during strain-path changes of magnesium alloy AZ31. International Journal of Plasticity 25, 861-880.

Simmons G., Wang H., 1971. Single Crystal Elastic Constants and Calculated Aggregate Properties, 2nd ed. M.I.T. Press, Cambridge, Mass.

Skippon T., Daymond M.R., On the prediction of self-consistent plasticity models: the effect of loading mode during in situ diffraction tests. Materials Science and Engineering A634, 77-85.

Spigarelli S., 2008. Constitutive equations in creep of Mg-Al alloys. Materials Science and Engineering A 492, 153-160.

Spigarelli S., El Mehtedi M., 2009. Microstructure-related equations for the constitutive analysis of creep in magnesium alloys. Scripta Materialia 61, 729-732.

Turner P.A., Tomé C.N., 1994. A study of residual stresses in Zircaloy-2 with rod texture. Acta Metallurgica et Materialia 42, 4143-4153. 
Ungar T., Mughrabi H., Ronnpagel D., Wilkens M., 1984. X-Ray line broadening study of the dislocation cell structure in deformed [001]-oriented copper single crystal. Acta Metallurgica 32, 333-342.

Wang H., Raeisinia B., Wu P.D., Agnew S.R., Tomé C.N., 2010a. Evaluation of self-consistent polycrystal plasticity models for magnesium alloy AZ31B sheet. International Journal of Solids and Structures 47, 2905-2917.

Wang H., Wu P.D., Neale K.W., 2010b. On the role of the constitutive model and basal texture on the mechanical behaviour of magnesium alloy AZ31B sheet. Journal of Zhejiang University SCIENCE A11, 744-755.

Wang H., Wu P.D., Tomé C.N., Huang Y., 2010c. A finite strain elastic-viscoplastic selfconsistent model for polycrystalline materials. Journal of the Mechanics and Physics of Solids 58, 594-612.

Wang H., Wu Y., Wu P.D., Neale K.W., 2010d. Numerical analysis of large strain simple shear and fixed-end torsion of HCP polycrystals. Computers Materials and Continua 19, 255284.

Wang H., Wu P.D., Tomé C.N., Wang J., 2012a. Study of lattice strains in magnesium alloy AZ31 based on a large strain elastic-viscoplastic self-consistent polycrystal model. International Journal of Solids and Structures 49, 2155-2167.

Wang H., Wu P.D., Tomé C.N., Wang J., 2012b. A constitutive model of twinning and detwinning for hexagonal close packed polycrystals. Materials Science and Engineering A555, 93-98.

Wang H., Clausen B., Tomé C.N., Wu P.D., 2013a. Studying the effect of stress relaxation and creep on lattice strain evolution of stainless steel under tension. Acta Materialia 61, 11791188.

Wang H., Wu P.D., Wang J., 2013b. Modeling inelastic behavior of magnesium alloys during cyclic loading-unloading. International Journal of Plasticity 47, 49-64.

Wang H., Wu P.D., Wang J., Tomé C.N., 2013c. A crystal plasticity model for hexagonal close packed crystals including twinning and de-twinning mechanisms. International Journal of Plasticity 49, 36-52.

Wenk H.R., Lutterotti L., Vogel S., 2003. Texture analysis with the new HIPPO TOF diffractometer. Nuclear Instruments and Methods in Physics Research A515, 575-588.

Wu L., Agnew S.R., Ren Y., Brown D.W., Clausen B., Stoica G.M., Fielden D.E., Wenk H.R., Liaw P.K., 2010. The Effects of Texture and Extension Twinning on the Low-cycle Fatigue Behavior of a Rolled Magnesium Alloy, AZ31B. Materials Science and Engineering A527, 7057-7067. 
Table title:

Table 1. Parameters associated with the dislocation density model.

Figure captions:

Figure 1. Initial texture of the AZ31 plate.

Figure 2. (a) Experimental stress strain curves along the TD of the plate; (b) holding times under tension with stress holds; (c) holding times under compression with stress holds; (d) holding times under compression with strain holds.

Figure 3. Schematic illustration of RSS distribution given by Eq. 2 using (a) $\bar{\tau}=25 M P a, V=$ $0.5 M P a$, (b) $\bar{\tau}=27 M P a, V=1 M P a$, and (c) $\bar{\tau}=29 M P a, V=2 M P a$.

Figure 4. Measured (symbols) and predicted (lines) stress strain curves of AZ31 plate under uniaxial tension and compression.

Figure 5. Relative activities of deformation mechanisms as a function of the accumulated strain. The measured (solid circles) and predicted (black solid line) twin volume fractions under compression are included.

Figure 6. The evolution of dislocation densities of deformation mechanisms of slip and the evolution of $\boldsymbol{V}$ under compression and tension.

Figure 7. Schematic representation of the evolution of RSS distribution during strain holding: (a) average RSS $\bar{\tau}$ - shear strain $\Gamma$ curve; (b) the RSS distribution at A; (c) the RSS distribution at B; (d) the RSS distribution at C.

Figure 8. Stress strain curves of AZ31 plate under compression and tension along the TD showing stress relaxation during strain holds.

Figure 9. Stress evolution with time during strain holding of AZ31 plate under compression and tension along the TD (notice different vertical scales).

Figure 10. Relative activities of deformation mechanisms for in-plane compression with strain holds as a function of (a) strain and (b) time; (c) detail of system activity during one loadunload-reload period at holding strain $\varepsilon=-0.06$.

Figure 11. Distribution of the dislocation density among grains at the start and end of relaxation at strains of (a) $6 \%$ and (b) $10 \%$ for the compression test with strain holds. 
Figure 12. Schematic representation of the evolution of RSS distribution during stress holding: (a) average RSS $\bar{\tau}$ vs shear strain $\Gamma$; (b) the RSS distribution at A; (c) the RSS distribution at B; (d) the RSS distribution at C.

Figure 13. (a) Engineering stress vs engineering strain curves of AZ31 plate under compression and tension with stress holds. (b) Comparison of engineering and true stress-strain curves for the same experiments.

Figure 14. Strain vs time curves for stress holds of AZ31 plate during compression and tension.

Figure 15. Lattice strain evolution of AZ31 plate under continuous compression, compression with stress holds and compression with strain holds.

Figure 16. Predicted lattice strain evolution of AZ31 plate under compression along TD during stress holdings and strain holdings. 
Table 1. Parameters associated with the dislocation density model

\begin{tabular}{|c|c|c|c|c|c|c|c|c|c|}
\hline & $\tau_{0}(M P a)$ & $\rho_{0}^{\alpha}\left(m^{-2}\right)$ & $b^{\alpha}(\AA)$ & $k_{1}(m)$ & $g^{\alpha}$ & $D^{\alpha}(M P a)$ & $C^{\alpha \beta}$ & $\chi$ & $n$ \\
\hline Prismatic & 98 & $1 \mathrm{e} 7$ & 3.21 & $8 \mathrm{e} 9$ & 0.033 & 80 & 1 & 0.2 & 13 \\
\hline Basal & 25 & $1 \mathrm{e} 7$ & 3.21 & $4 \mathrm{e} 8$ & 0.033 & 80 & 1 & 0.2 & 13 \\
\hline Pyramidal & 315 & $1 \mathrm{e} 7$ & 6.12 & $1.2 \mathrm{e} 8$ & 0.033 & 80 & 1 & 0.2 & 13 \\
\hline Twinning & 35 & NA & 0.492 & NA & NA & NA & NA & NA & 13 \\
\hline
\end{tabular}




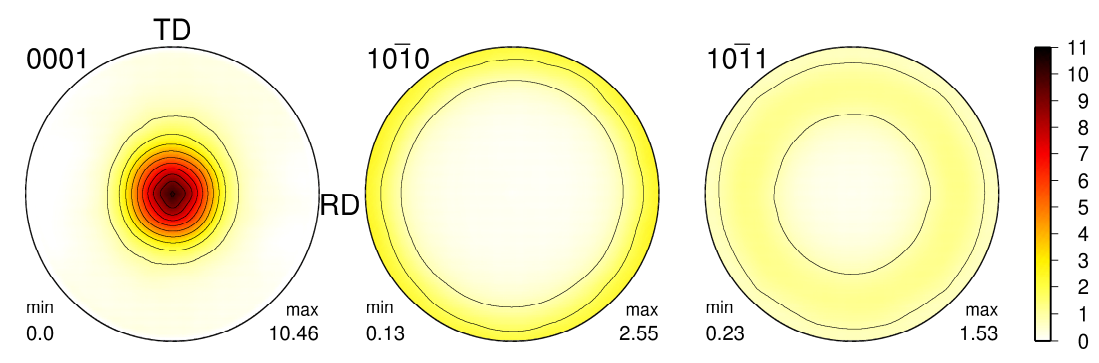

Figure 1. Initial texture of the AZ31 plate.
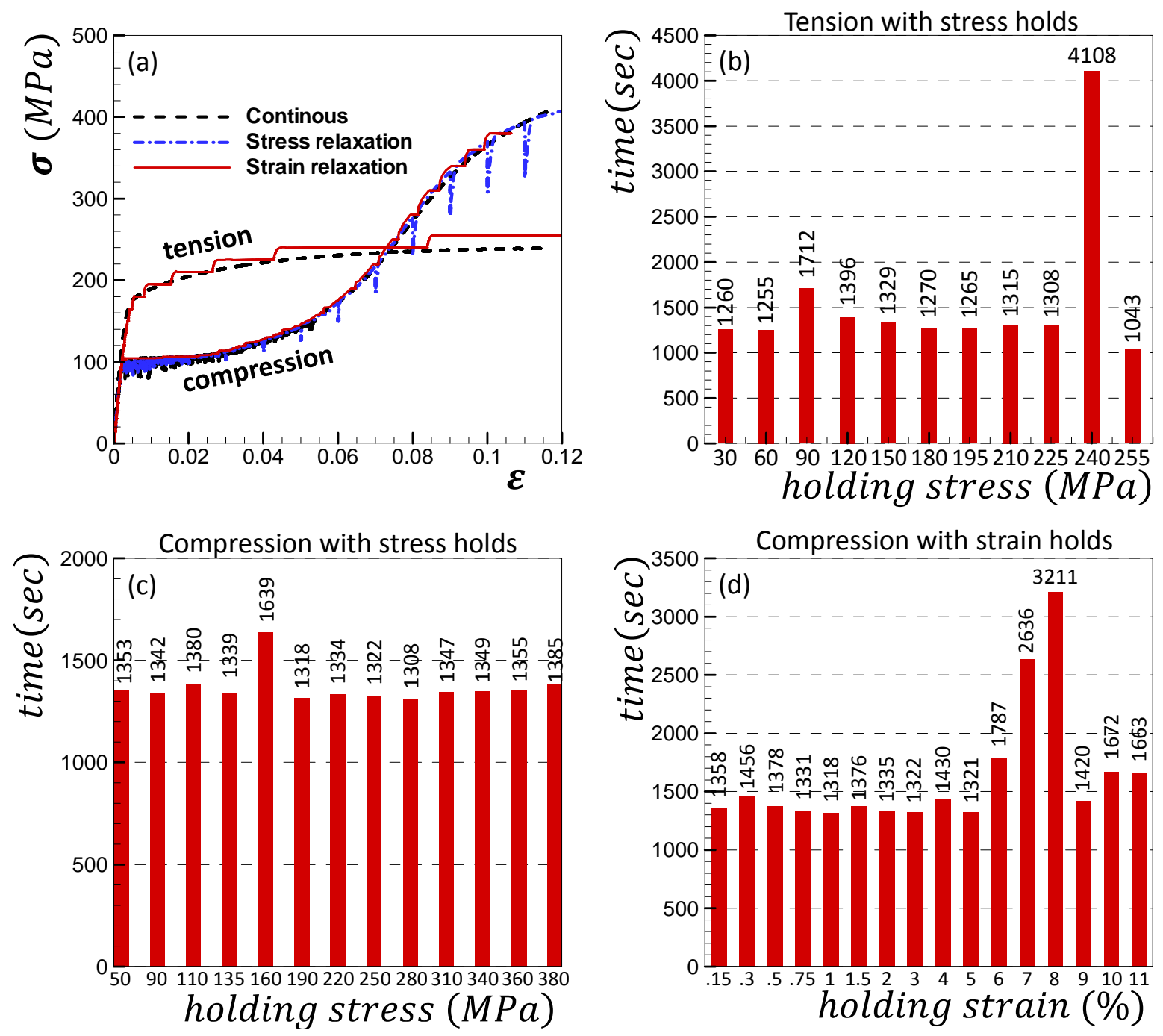

Figure 2. (a) Experimental stress strain curves along the TD of the plate; (b) holding times under tension with stress holds; (c) holding times under compression with stress holds; (d) holding times under compression with strain holds. 

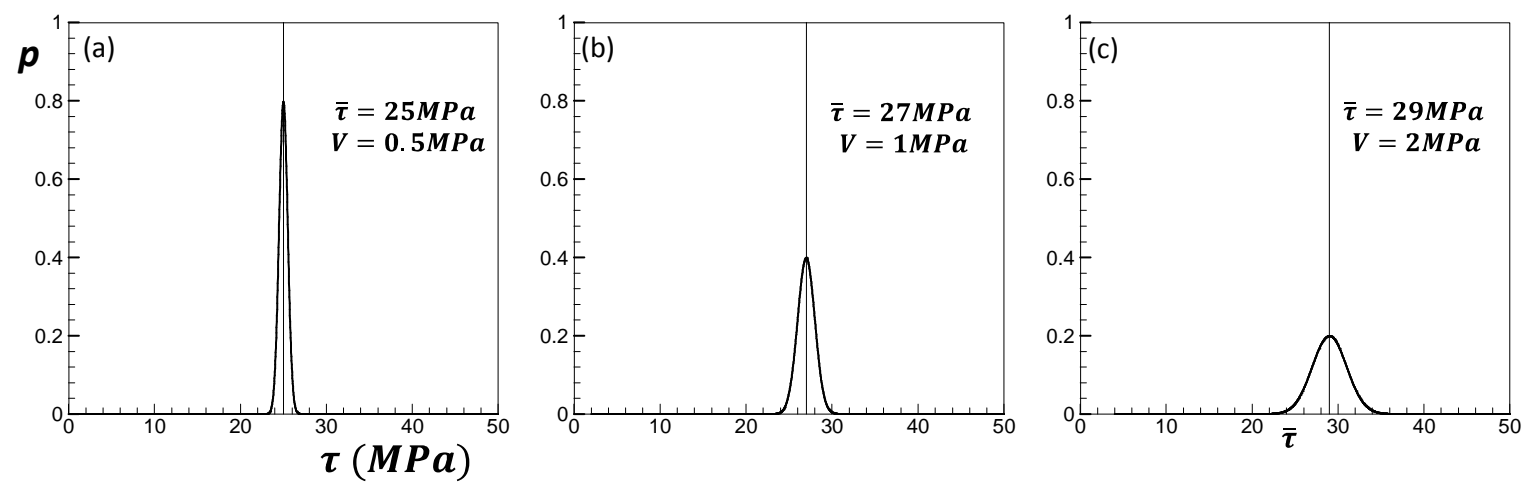

Figure 3. Schematic illustration of RSS distribution given by Eq. 2 using (a) $\bar{\tau}=25 M P a, V=$ $0.5 M P a$, (b) $\bar{\tau}=27 M P a, V=1 M P a$, and (c) $\bar{\tau}=29 M P a, V=2 M P a$.

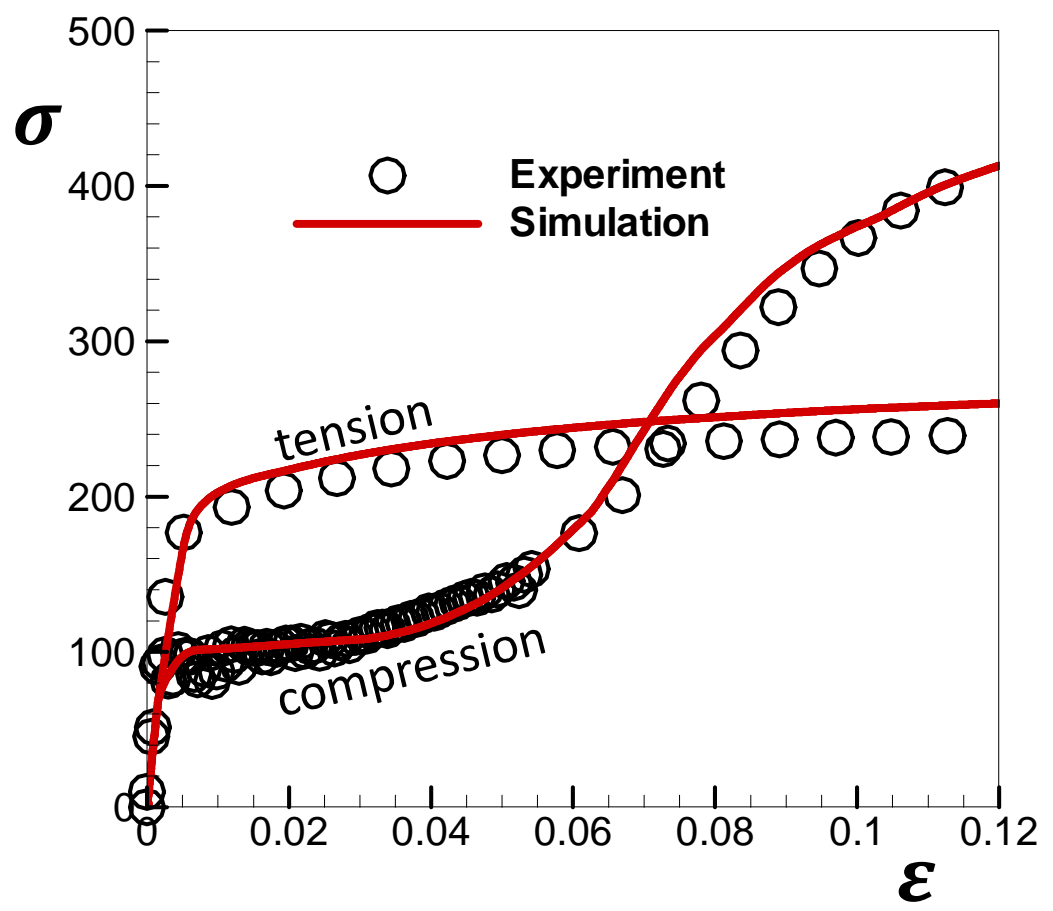

Figure 4. Measured (symbols) and predicted (lines) stress strain curves of AZ31 plate under uniaxial tension and compression. 

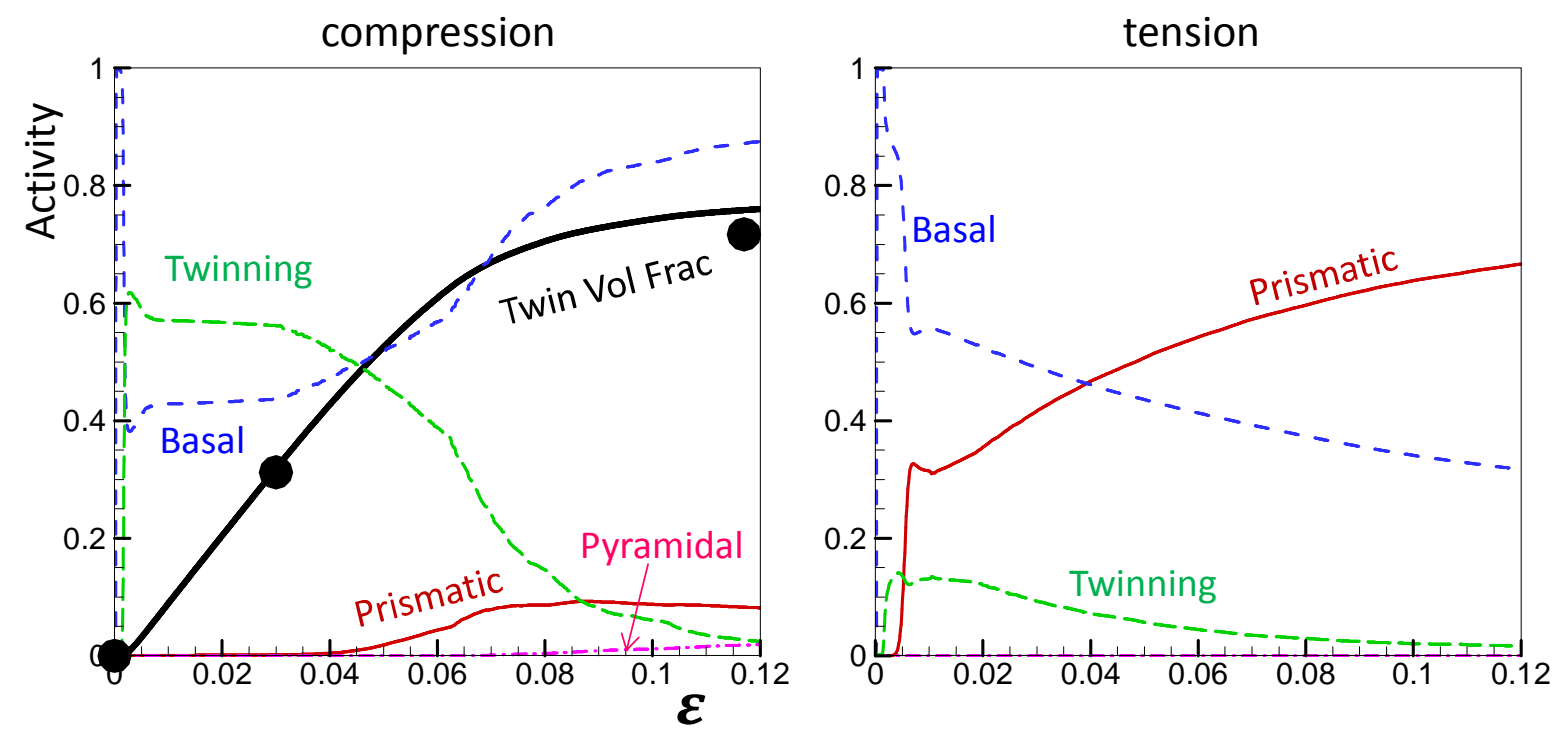

Figure 5. Relative activities of deformation mechanisms as a function of the accumulated strain.

The measured (solid circles) and predicted (black solid line) twin volume fractions under compression are included.
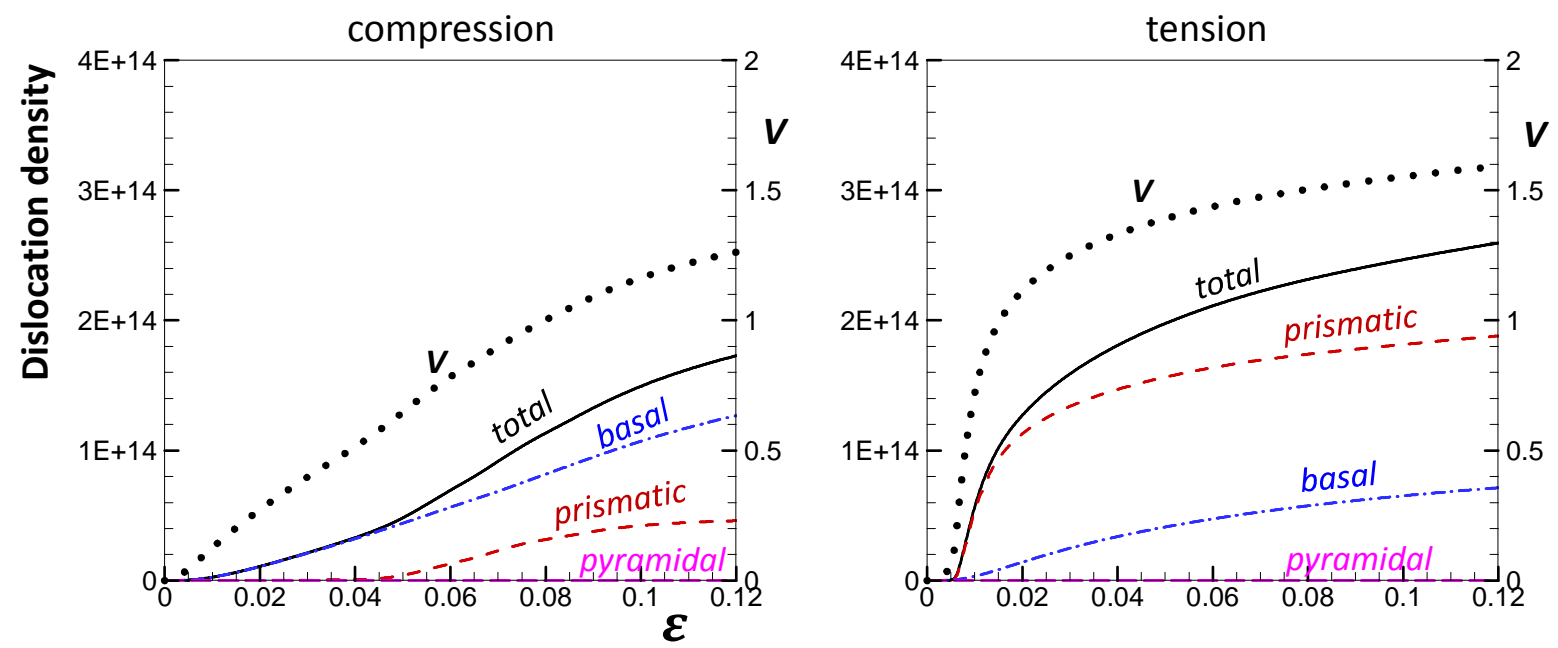

Figure 6. The evolution of dislocation densities of deformation mechanisms of slip and the evolution of $\boldsymbol{V}$ under compression and tension. 

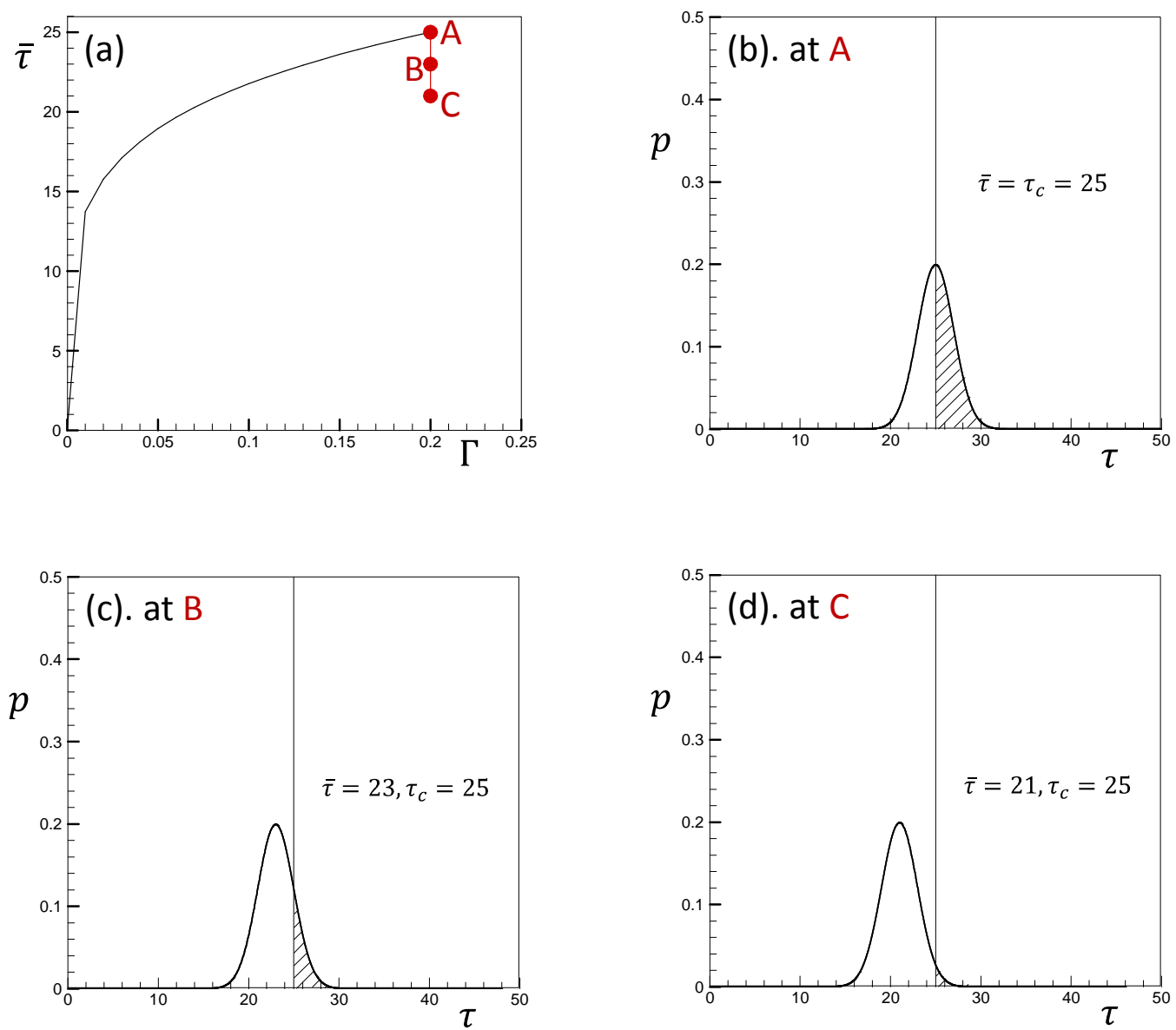

Figure 7. Schematic representation of the evolution of RSS distribution during strain holding: (a) average RSS $\bar{\tau}$ - shear strain $\Gamma$ curve; (b) the RSS distribution at A; (c) the RSS distribution at B; (d) the RSS distribution at C. 


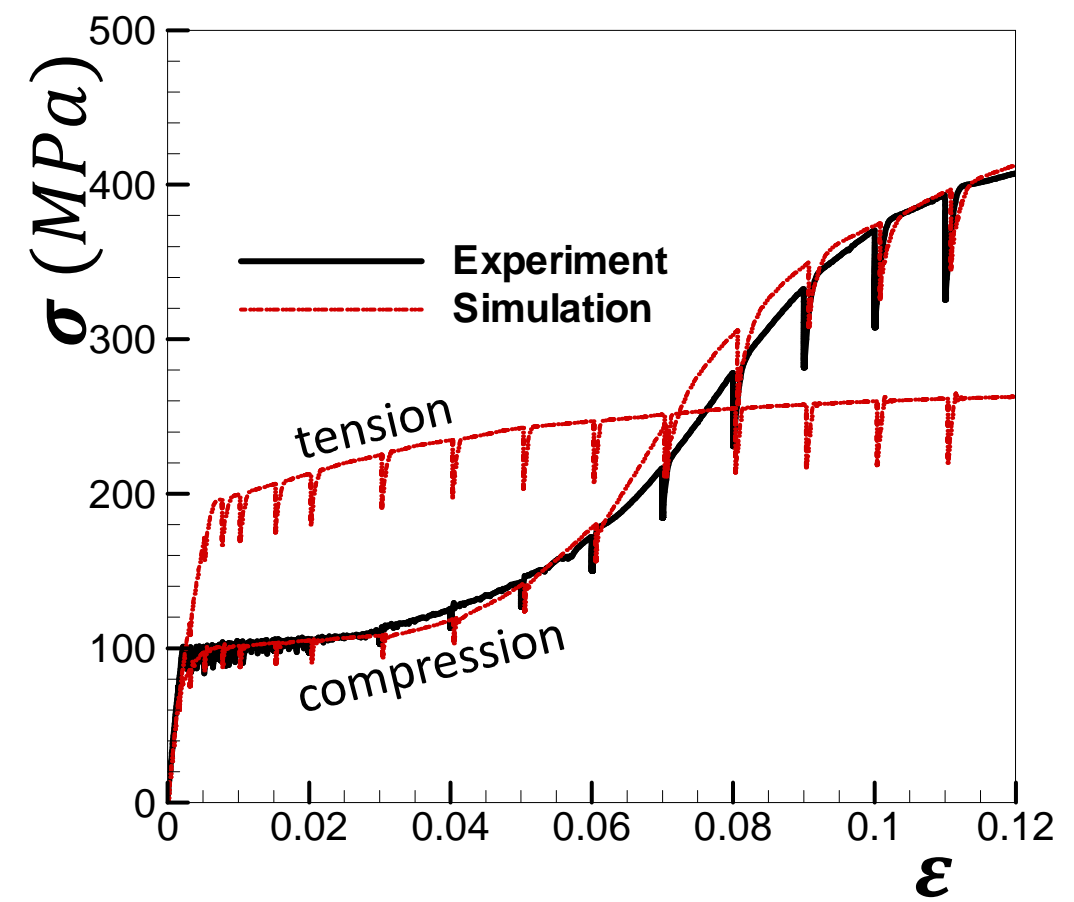

Figure 8. Stress strain curves of AZ31 plate under compression and tension along the TD showing stress relaxation during strain holds.
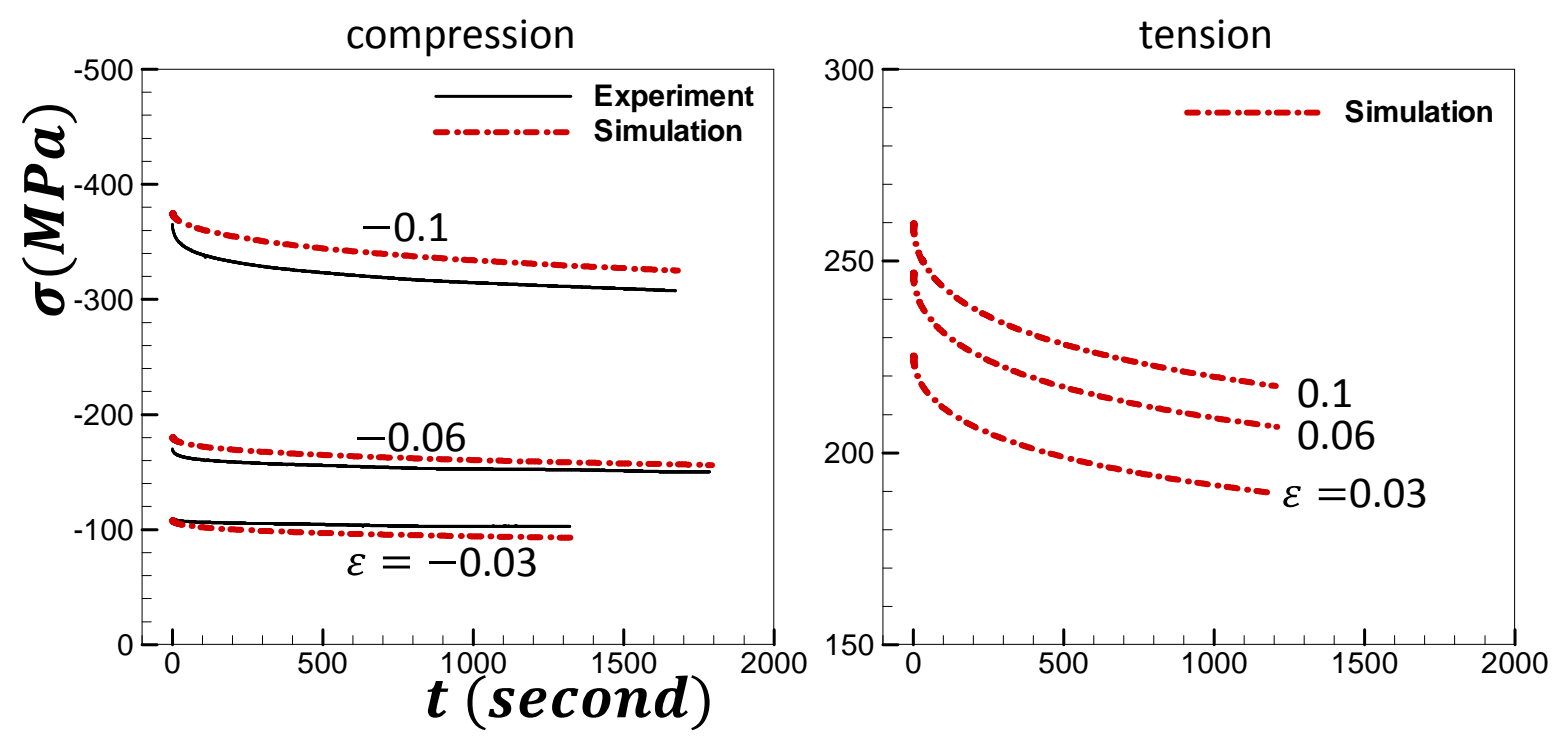

Figure 9. Stress evolution with time during strain holding of AZ31 plate under compression and tension along the TD (notice different vertical scales). 

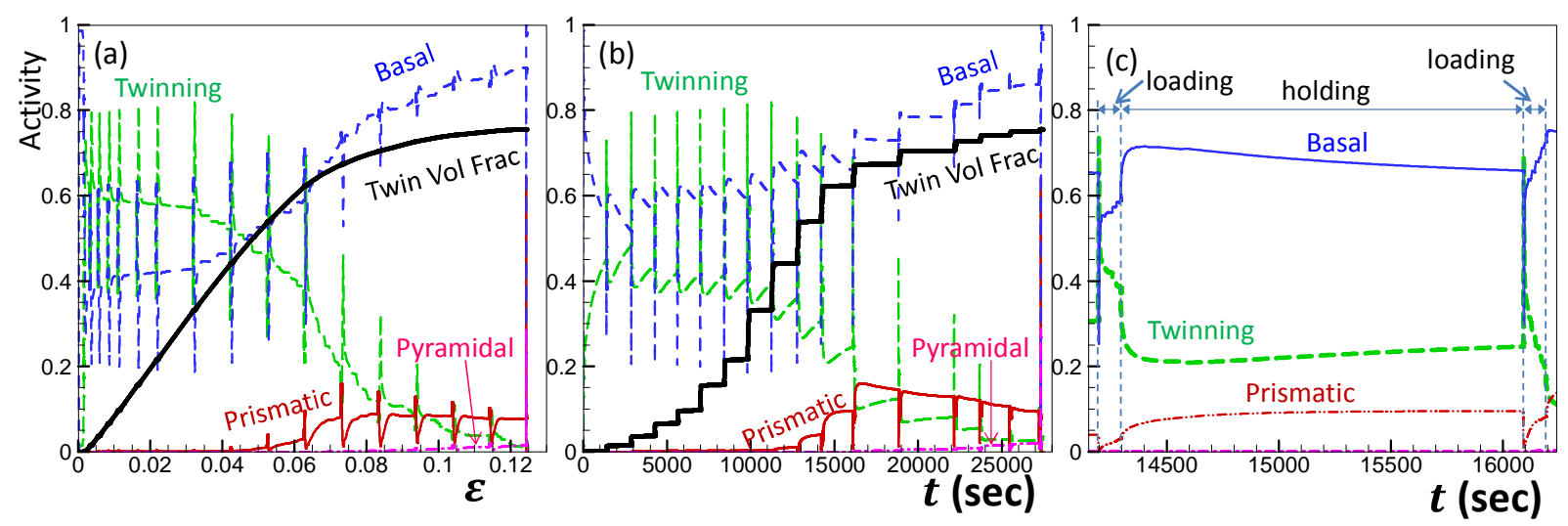

Figure 10. Relative activities of deformation mechanisms for in-plane compression with strain holds as a function of (a) strain and (b) time; (c) detail of system activity during one load-holdreload period at holding strain $\varepsilon=-0.06$.
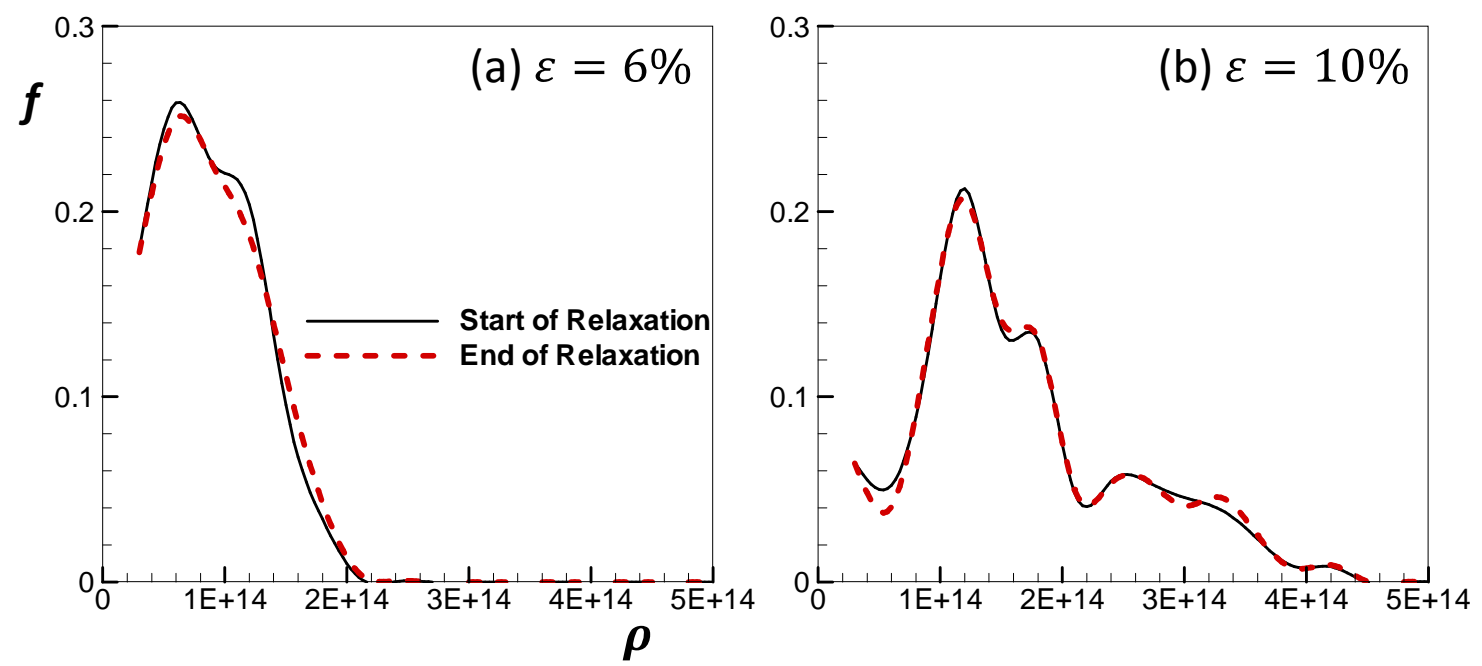

Figure 11. Distribution of the dislocation density among grains at the start and end of relaxation at strains of (a) $6 \%$ and (b) $10 \%$ for the compression test with strain holds. 

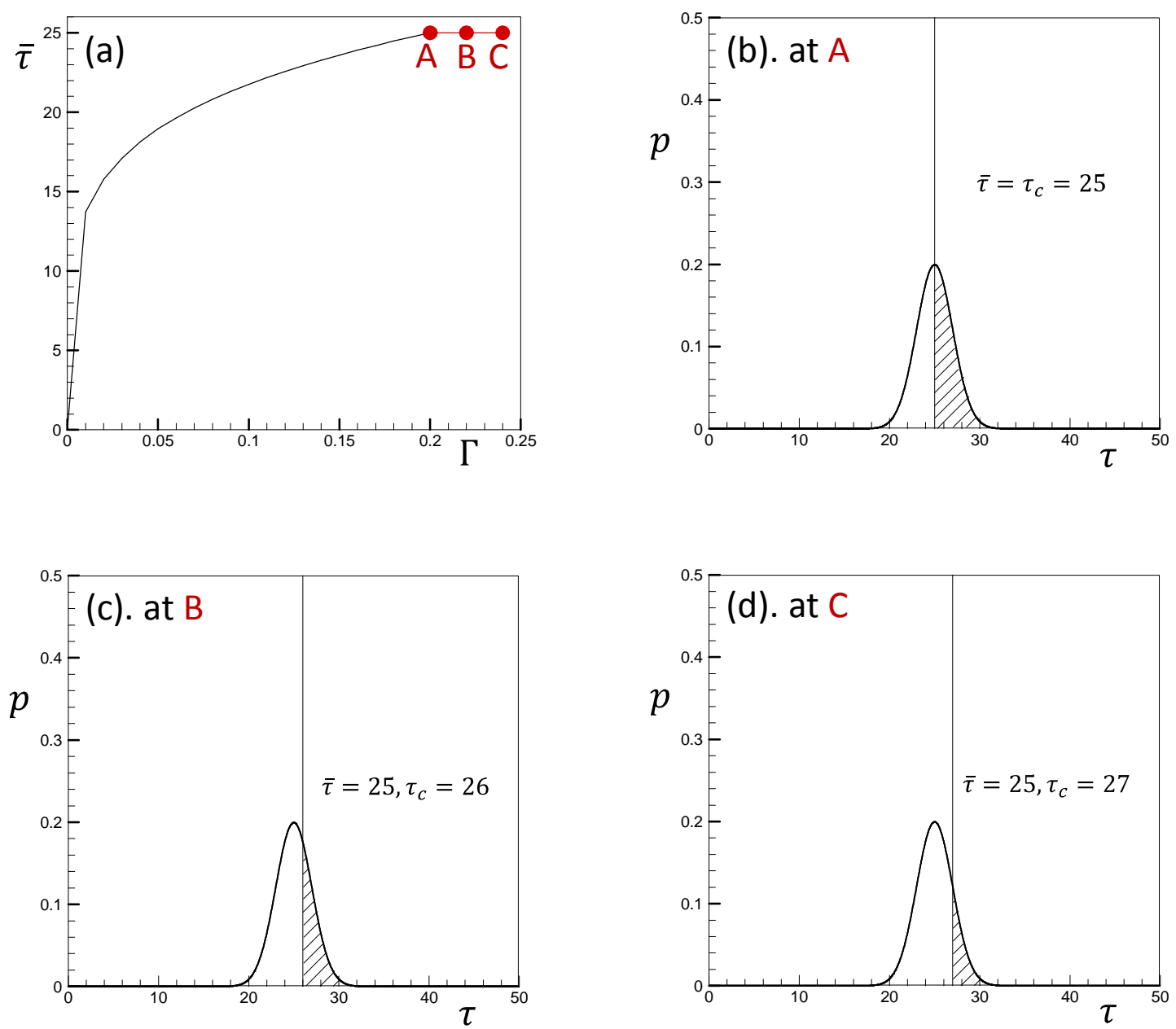

Figure 12. Schematic representation of the evolution of RSS distribution during stress holding: (a) average RSS $\bar{\tau}$ vs shear strain $\Gamma$; (b) the RSS distribution at A; (c) the RSS distribution at B; (d) the RSS distribution at $\mathrm{C}$. 

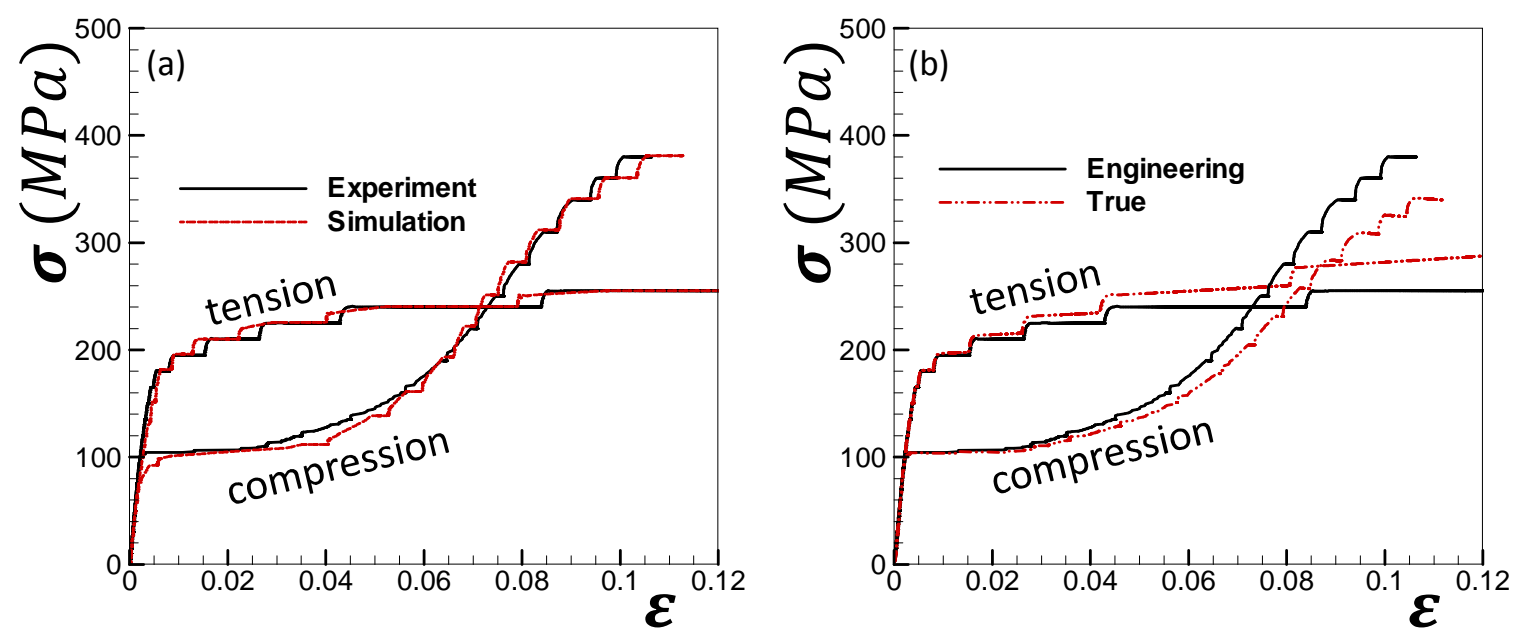

Figure 13. (a) Engineering stress vs engineering strain curves of AZ31 plate under compression and tension with stress holds. (b) Comparison of engineering and true stress-strain curves for the same experiments.
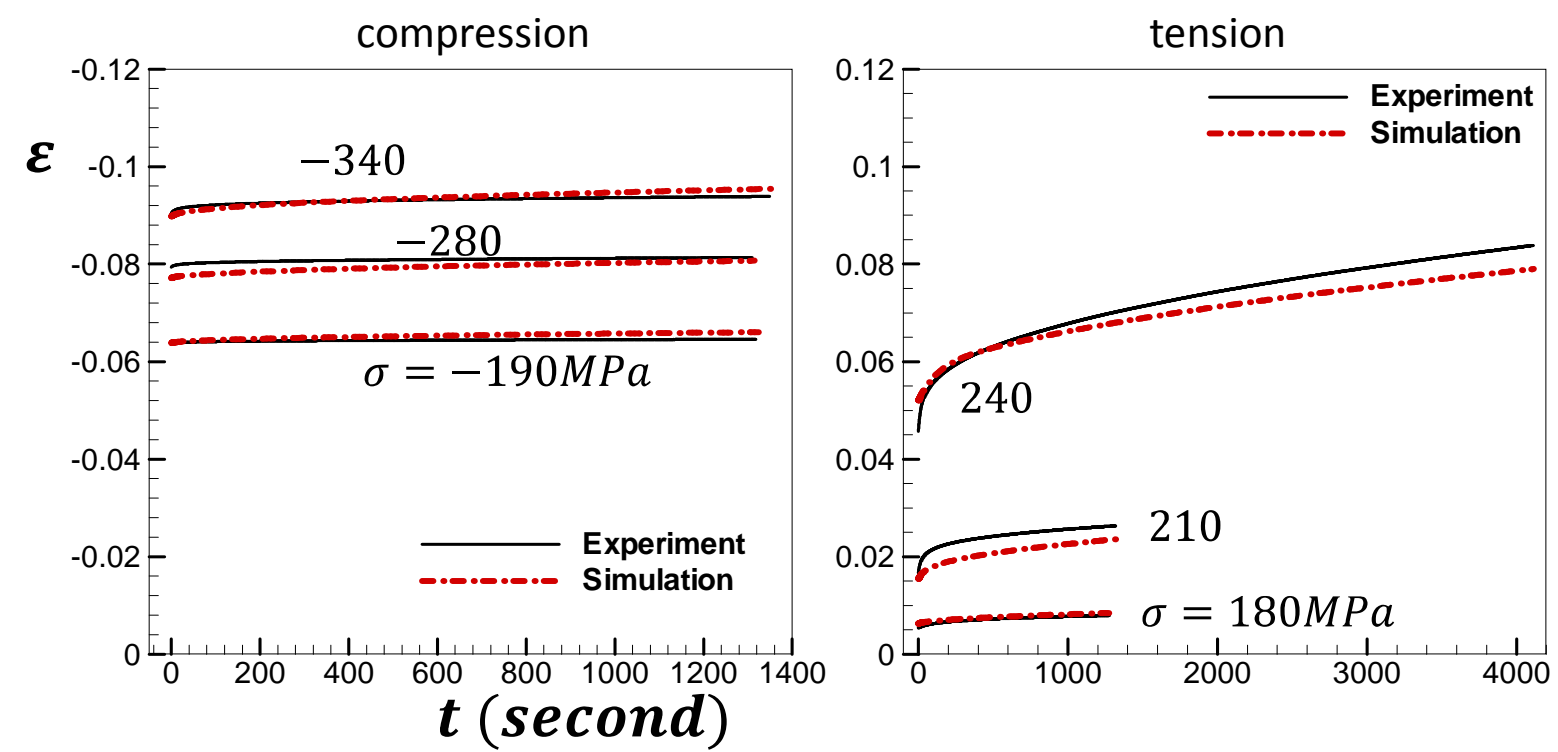

Figure 14. Strain vs time curves for stress holds of AZ31 plate during compression and tension. 

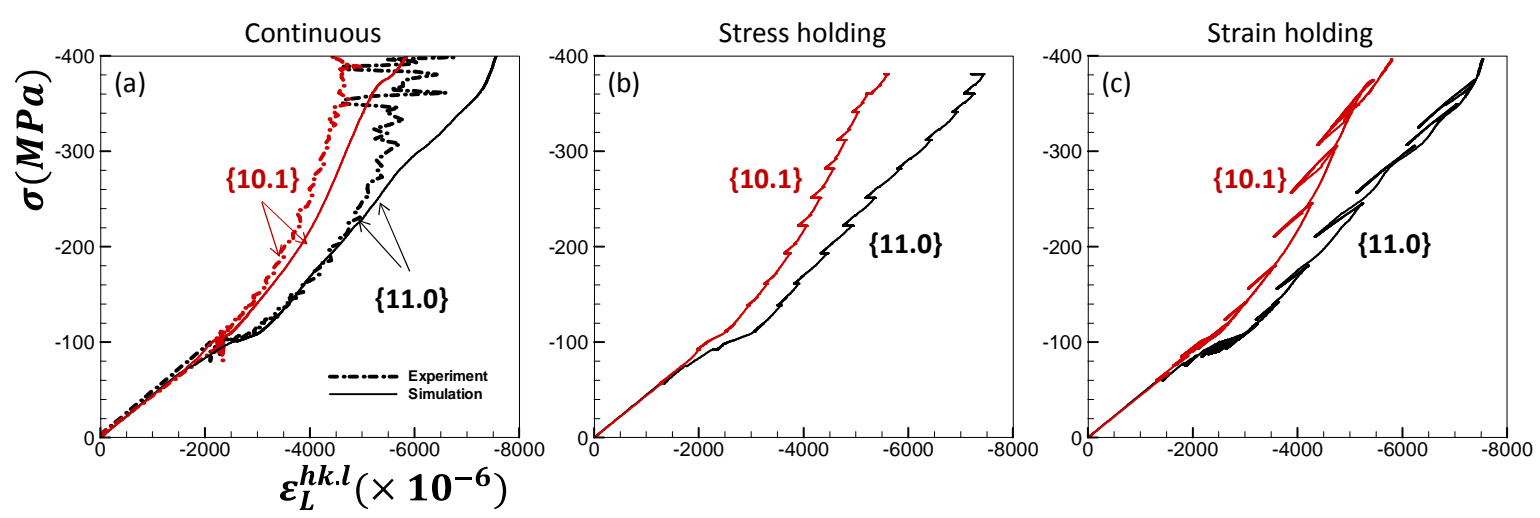

Figure 15. Lattice strain evolution of AZ31 plate under continuous compression, compression with stress holds and compression with strain holds.
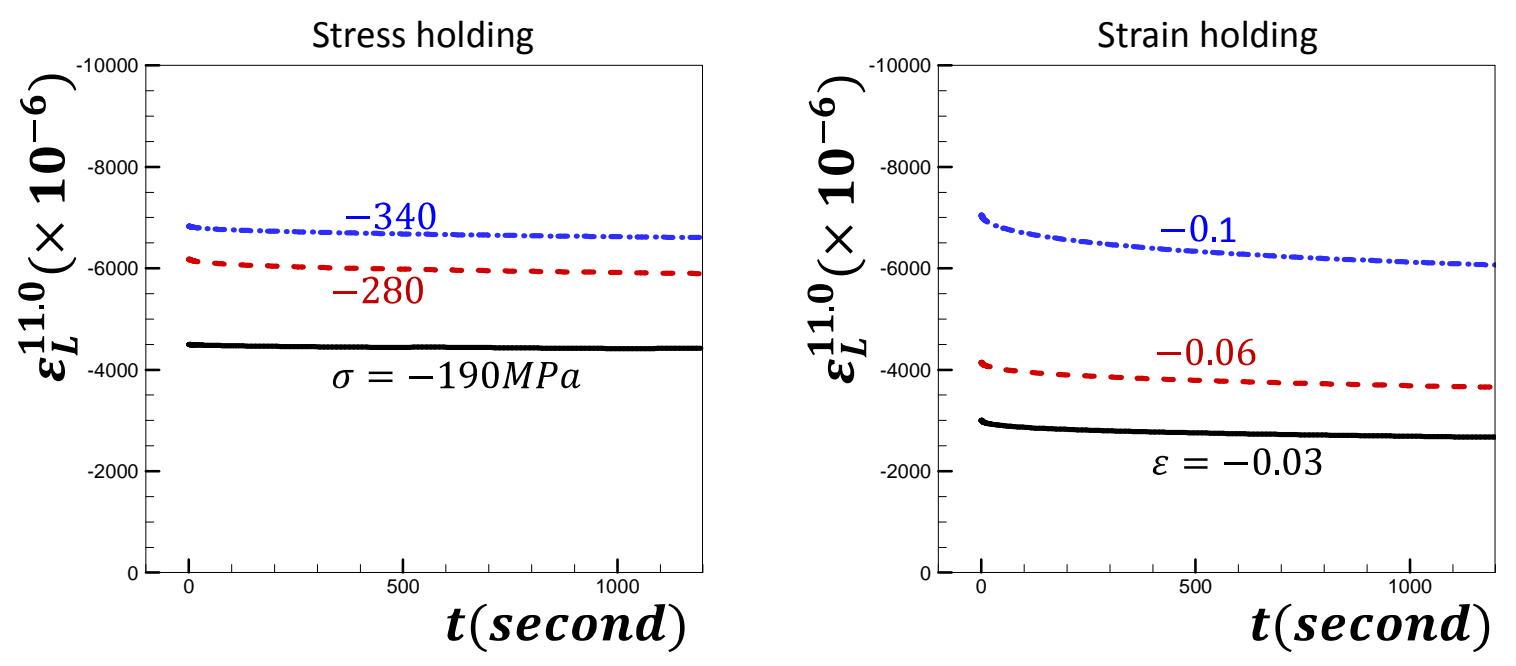

Figure 16. Predicted lattice strain evolution of AZ31 plate under compression along TD during stress holdings and strain holdings. 